\title{
Effects of Active and Passive Control Techniques on Mach 1.5 Cavity Flow Dynamics
}

\author{
Selin Aradag, Kubra Asena Gelisli, and Elcin Ceren Yaldir \\ Department of Mechanical Engineering, TOBB University of Economics and Technology, Sogutozu Cad. No. 43, 06560 Ankara, Turkey \\ Correspondence should be addressed to Selin Aradag; selinaradag@gmail.com
}

Received 27 December 2016; Revised 30 March 2017; Accepted 20 April 2017; Published 25 May 2017

Academic Editor: Mahmut Reyhanoglu

Copyright (C) 2017 Selin Aradag et al. This is an open access article distributed under the Creative Commons Attribution License, which permits unrestricted use, distribution, and reproduction in any medium, provided the original work is properly cited.

\begin{abstract}
Supersonic flow over cavities has been of interest since 1960s because cavities represent the bomb bays of aircraft. The flow is transient, turbulent, and complicated. Pressure fluctuations inside the cavity can impede successful weapon release. The objective of this study is to use active and passive control methods on supersonic cavity flow numerically to decrease or eliminate pressure oscillations. Jet blowing at several locations on the front and aft walls of the cavity configuration is used as an active control method. Several techniques are used for passive control including using a cover plate to separate the flow dynamics inside and outside of the cavity, trailing edge wall modifications, such as inclination of the trailing edge, and providing curvature to the trailing edge wall. The results of active and passive control techniques are compared with the baseline case in terms of pressure fluctuations, sound pressure levels at the leading edge, trailing edge walls, and cavity floor and in terms of formation of the flow structures and the results are presented. It is observed from the results that modification of the trailing edge wall is the most effective of the control methods tested leading to up to $40 \mathrm{~dB}$ reductions in cavity tones.
\end{abstract}

\section{Introduction}

Supersonic flow over cavities has been a fluid dynamics phenomenon of interest since the 1960s. Cavities represent the models of bomb bays of aircraft; therefore, they have been crucial for aerodynamic applications. Supersonic aircraft carry the stores inside and the velocity must be decreased to subsonic zone during store separation. This leads to more time over the target leading to increased possibility of being detected by a radar. Carrying the stores outside of the aircraft is a possible solution. However, this increases the radar signature, as well as the aerodynamic loads and heating. Therefore, it is important for supersonic aircraft to carry the stores inside and to be able to release them at supersonic speeds.

Supersonic cavity flow has gained importance due to these necessities. Although performing experiments at these speeds requires specifically designed wind tunnels, the advent of the computational techniques and computer capabilities with the help of computer clusters increased interest towards this flow. The flow is three-dimensional, turbulent, and very complicated in nature due to its high speed, different modes of vortical phenomenon, and high speed waves.

There are several studies in literature that explain the supersonic flow structure inside the cavities based on rigorous experiments or time consuming computations. It can be said that the cavity dynamics is now known and well documented and can be explained by combining several fluid dynamics phenomenon, although it is complicated and random in nature [1]. The pressure fluctuations inside the cavity are very high, the peaks can be three times the mean pressure and this increases the sound pressure levels in the cavity which may cause the unsuccessful release of the stores. In some extreme cases, the stores can return and hit the aircraft.

The next step in cavity flow research is the application of new or established control techniques on the flow structure and to observe the effects of these techniques on the flow, in terms of pressure fluctuations and sound pressure levels. If the pressure fluctuations can be eliminated or at least suppressed, this can be a meaningful step on the road to supersonic era. There are several studies in literature on different active 
and passive control techniques to suppress these pressure oscillations. Lawson and Barakos present a review of numerical simulations for high speed, turbulent cavity flows [2]. Several experimental and numerical control techniques are to decrease the pressure oscillations inside the cavity and are summarized in their review. Cattafesta III et al. also provide a review on active control techniques for pressure oscillations in the cavities focusing on practical applications [3]. Rizzetta et al. [4] performed Large Eddy Simulations (LES) of supersonic cavity flow, including the simulations with applied control techniques. They also provided comparisons with experimental data where available. Comte et al. carried out simulations of high-subsonic cavity flow controlled with a spanwise rod [5]. Levasseur et al. [6] performed LES of flow on unstructured meshes by implementing passive control techniques on subsonic cavities, as well as experiments. After validation of the baseline case with experiments, they concentrated on two passive acoustic flow control methods: rod in cross-flow and flat-top spoiler. Leading edge blowing was proved to be useful for the alleviation of cavity loads by Arunajatesan et al. [7]. Aradag et al. [8], Yilmaz and Aradag [9], and Lazar et al. [10] concentrated on localized laser energy deposition for active cavity flow control. Another interesting passive control technique proposed by Lee et al. [11] is a subsonic cavity installed at the leading edge of the supersonic cavity. They also worked on the determination of the effects of a triangular bump on the oscillations.

Experimental studies of flow control for supersonic cavities have also been a topic of interest for several years. For example, Ukeiley et al. [12] worked on control of cavity flow using active fluid injection methods and passive modification methods for cavity walls. Alvi and Cattafesta III [13] investigated the effects of zero-net mass-flux actuators on several types of flow including the flow over the cavity. The power of using smoke, shadowgraph, Schlieren images, and Particle Image Velocimetry is investigated. Zhuang et al. used supersonic microjets for Mach 2 cavity flow control [14]. Flow control using plasma actuators was also investigated for supersonic cavities by Narayanaswamy et al. [15]. Another research group who worked on control of supersonic cavity oscillations by microjet injection is Thangamani and Kurian [16]. They observed in their experiments that microjets reduce the coherence of the shear layer but increase the turbulence.

In this research, the objective is to suppress pressure oscillations caused by the supersonic flow over cavities with the help of active and passive control techniques. Jet blowing at several locations on the front and aft walls of the cavity configuration is used as an active control method. Several techniques are used for passive control including, using a cover plate to separate the flow dynamics inside and outside of the cavity, trailing edge wall modifications, such as inclination of the trailing edge, and providing curvature to the trailing edge wall. The results of active and passive control techniques are compared with the baseline case in terms of pressure fluctuations both on the trailing wall and on the cavity floor, sound pressure levels at the leading edge, trailing edge walls, and cavity floor and in terms of formation of the flow structures as presented by density, pressure, temperature, streamwise velocity contours, and streamlines.
TABLE 1: Numerical parameters.

\begin{tabular}{lc}
\hline Total pressure & $66.4 \mathrm{kPa}$ \\
Total temperature & $218 \mathrm{~K}$ \\
Mach number & 1.5 \\
Reynolds number & $1.09 \times 10^{6}$ \\
Cavity length & $0.012065 \mathrm{~m}$ \\
Cavity depth & $0.0238 \mathrm{~m}$ \\
Cavity width & $0.0635 \mathrm{~m}$ \\
Boundary layer thickness & $0.0051 \mathrm{~m}$ \\
\hline
\end{tabular}

\section{Methodology}

Baseline case used in the computations is taken from the experiments of Kaufman et al. [17]. Length to depth ratio of the cavity is 5.07 and the Mach number is 1.5 . Table 1 shows the parameters used in the CFD analysis.

A representative sketch of the geometry of the cavity under consideration is shown in Figure 1(a). Figure 1(b) shows the geometry for the computations with the dimensions in millimeters.

The details of the baseline computations are given in a previous study of our group [18]. The computations are performed using commercial Ansys Fluent software. For time resolution, second-order implicit method is used with 20 inner iterations for each time step. Second-order upwind method is used for space discretization. Density based algorithm is utilized since the flow is compressible. $k-\omega$ twoequation turbulence model is used in Reynolds averaged Navier-Stokes (RANS) equations to obtain the transient, turbulent flow characteristics.

Outlet and upper wall boundary conditions are selected as pressure far field, and these boundaries are placed far enough to avoid reflection. No slip wall boundary condition is used for the cavity walls. The solution of the two-dimensional steady-state Navier-Stokes equations over a flat plate is used as inflow boundary condition to make sure the flow is fully developed at the entrance of the cavity. Boundary layer thickness of the incoming flow matches the experimental values. Per Kaufman et al.s experiments [17], the boundary layer thickness is $0.0051 \mathrm{~m}$. The boundary layer thickness used as the inlet boundary condition matches the experiments as shown in Figure 2.

Flow characteristics for the baseline case (Mach 1.5 supersonic cavity flow with $L / D=5.07$ ) are shown in Figure 3. Cavity flow is very complex in nature, comprising vortexwall, shear layer-wall, and shock wave interactions causing pressure fluctuations. The flow is transient, however periodic in nature. The period of the fluctuations is called "Rossiter period" named after the pioneer of the cavity flow, Rossiter [19]. The trailing edge wall of the cavity witnesses more pressure fluctuations and higher sound pressure levels (SPL) because of the wall-shear layer interactions.

The following flow phenomena are observed in one Rossiter period of the periodic cavity flow:

(1) The free stream flow separates inside the cavity and reattaches at the trailing edge wall. 


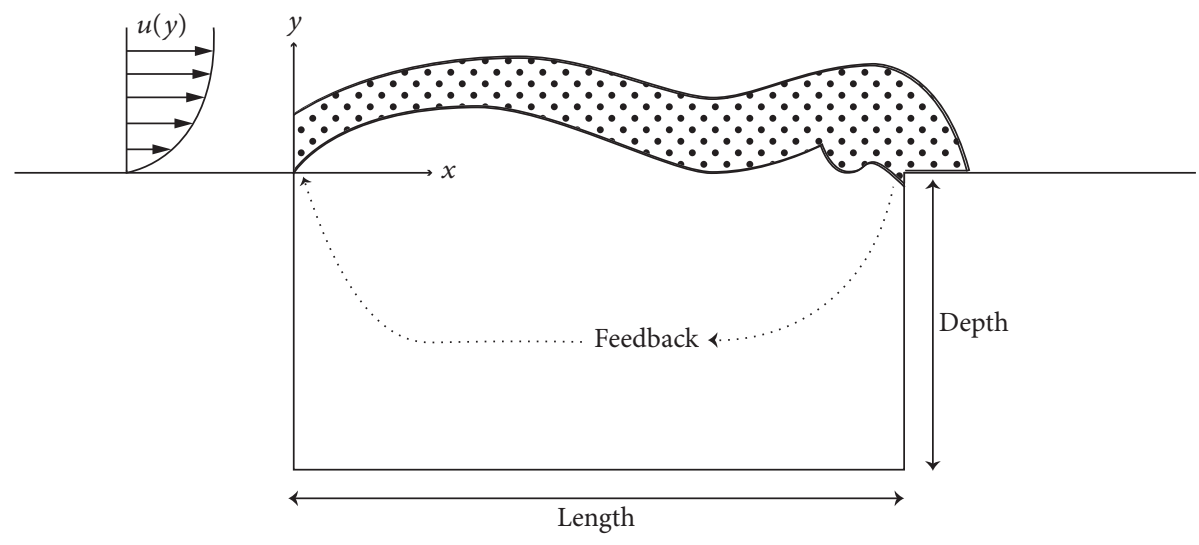

(a)

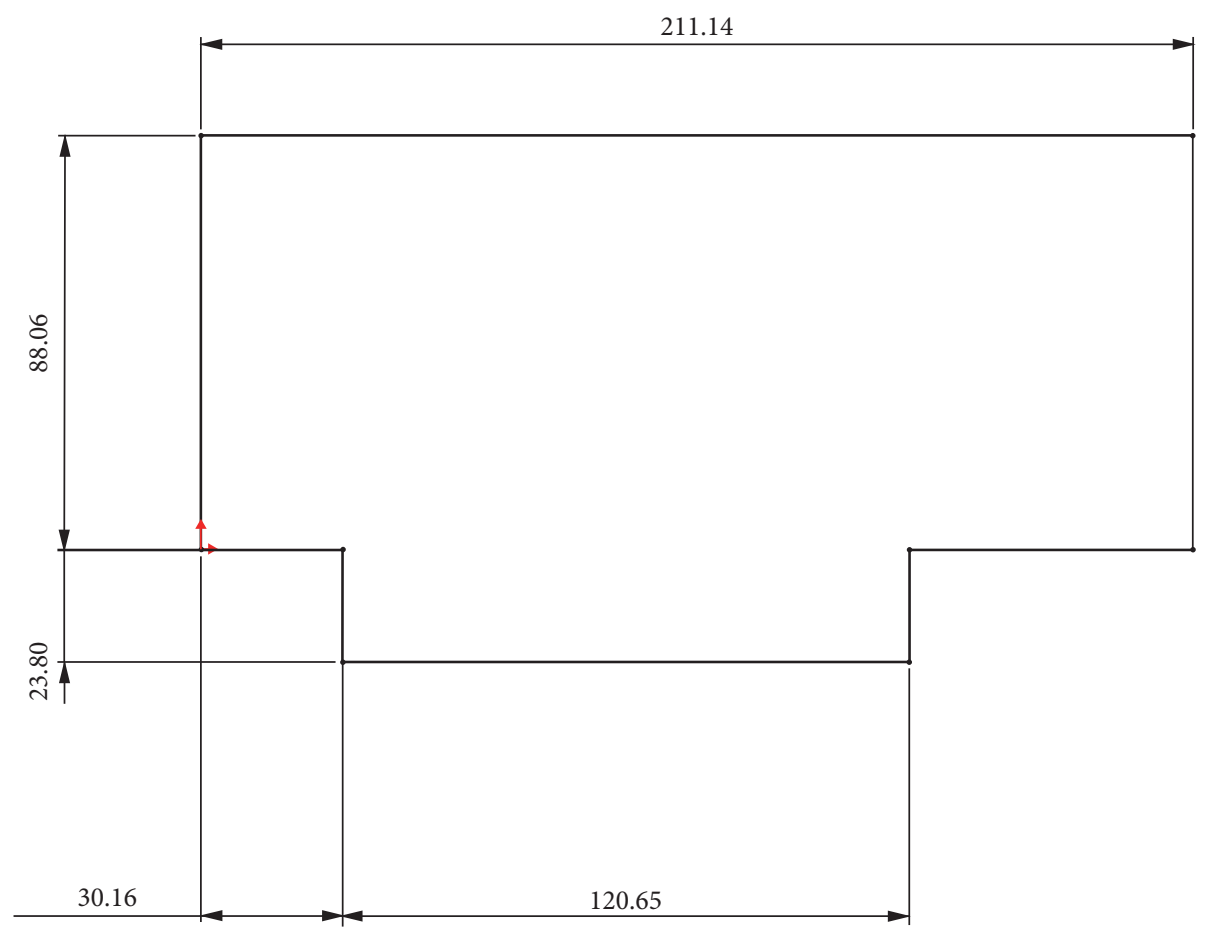

(b)

Figure 1: (a) Schematic view of the cavity. (b) Dimensions of the numerical domain.

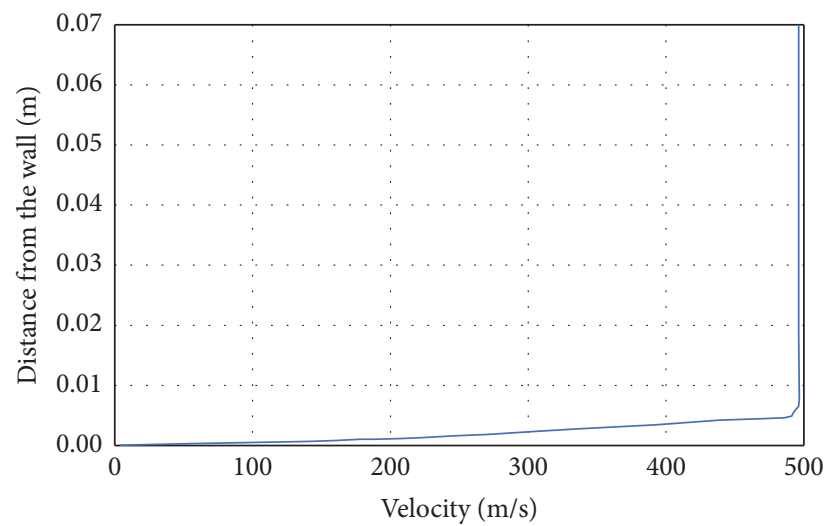

FIGURE 2: Boundary layer velocity profile used for the inlet boundary condition. 


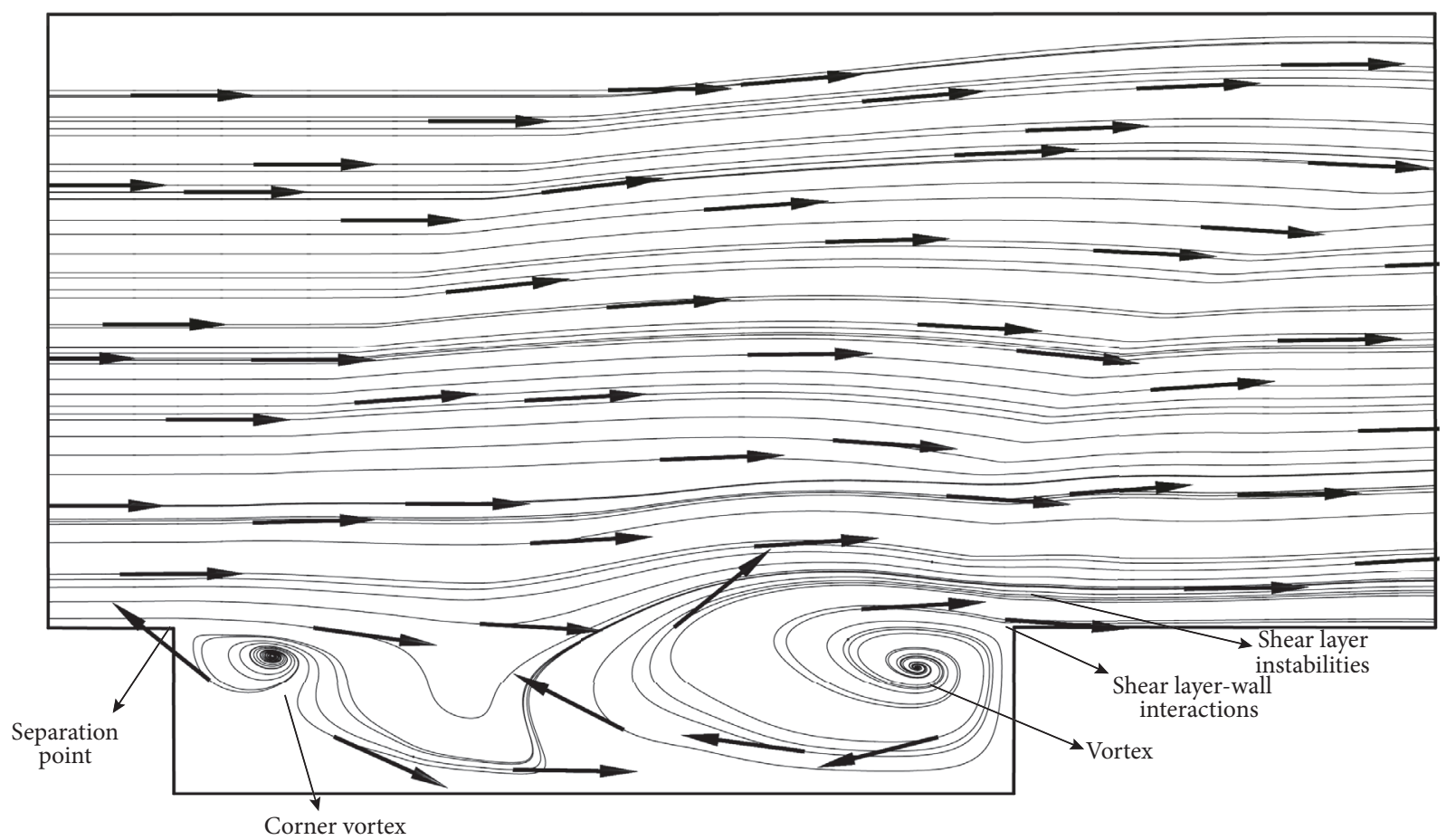

FIGURE 3: Supersonic cavity flow dynamics.

(2) The shear layer reattaches with the flow from inside the cavity and this causes vortices inside the cavity.

(3) The pressures inside the cavity decrease because of the vortices and the shear layer is bended and mass leaves the inside of the cavity.

(4) The mass transfer causes pressure fluctuations at the trailing wall of the cavity.

(5) The flow reaches back to the leading wall of the cavity and the pressures inside the cavity rise and mass is transferred to the outside zone.

Time step in the computations is 1 microsecond and the computations are performed for 14 periods corresponding to 12,000 time steps. A time step of 10 microseconds is also used and the results are compared with the results for a time step of 1 microsecond to select the proper time step. It is seen from the results that the periodic nature of the flow cannot be observed with a time step of 10 milliseconds. The pressures do not fluctuate, which is not physical.

Grid refinement study is also an essential part of this work as for all computational research. Six different grids are generated and the results are compared to each other and the experimental data of Kaufman et al. [17] to select the proper grid to be used in further flow control simulations. Table 2 shows the properties of the computational meshes used in the study.

If the pressure fluctuations can be eliminated or at least suppressed, this can be a meaningful step for flow control research. Passive and active flow control techniques are applied to the flow to decrease the pressure fluctuations and
TABLE 2: The properties of the computational meshes used in the study.

\begin{tabular}{lccc}
\hline Grid & Number of grid cells & Average $y+$ & Minimum $y+$ \\
\hline 1 & 4055 & 41.8 & 3.31 \\
2 & 16104 & 11.9 & 0.31 \\
3 & 36321 & 3.4 & 0.07 \\
4 & 54721 & 3.0 & 0.05 \\
5 & 64410 & 3.1 & 0.03 \\
6 & 337356 & 1.21 & $<0.001$ \\
\hline
\end{tabular}

consequently the sound pressure levels (SPL) inside and on the walls of the cavity to enable successful store release. The advantage of passive control techniques is the fact that they are simple and inexpensive. However, once they are applied to the flow, they cannot be changed when it is necessary, since they include geometry modifications. Active control techniques are applied in real time only when it is necessary; that is, the flow structure needs to be modified. At other times, they can be turned off, which makes them expensive but powerful. Jet blowing through four different locations and the combinations of these four locations are the active flow control method utilized. In terms of passive control, using a cover plate to separate the flow dynamics inside and outside of the cavity, trailing edge wall modifications, such as inclination of the trailing edge (with different slopes), and providing curvature to the trailing edge wall are used. The test cases for flow control are summarized in Table 3. Figure 4 shows these modifications on a sketch for clarity. 


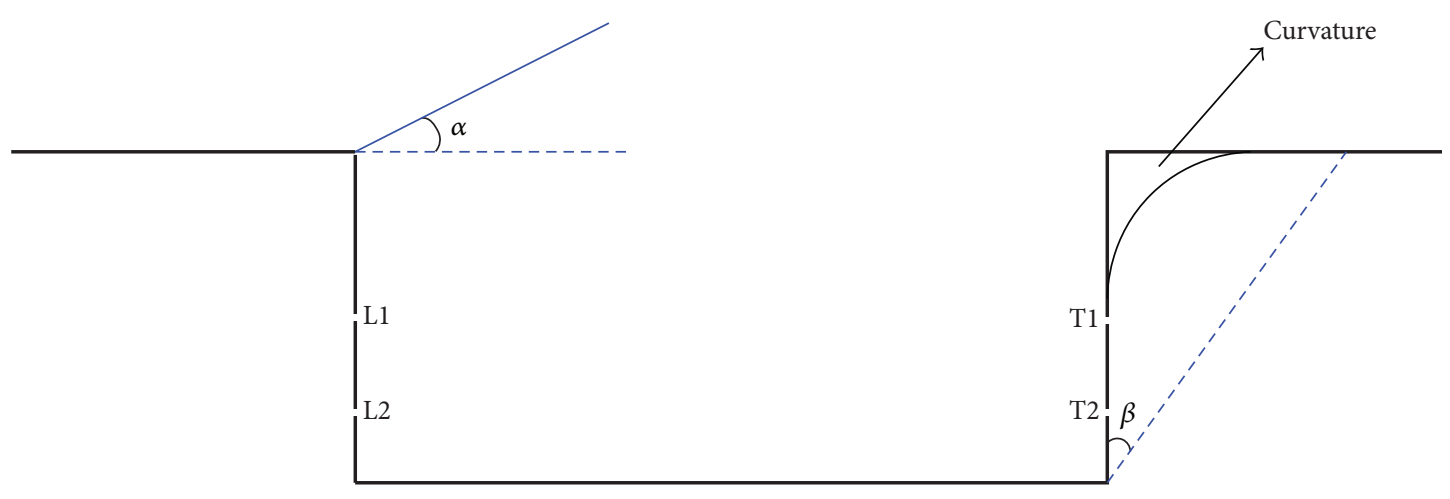

FIGURE 4: Modifications for passive and active flow control.

TABLE 3: Active and passive control cases.

\begin{tabular}{|c|c|c|c|c|c|}
\hline Control method & Case 1 & Case 2 & Case 3 & Case 4 & Case 5 \\
\hline Trailing edge wall modification (TWM) & $\beta=30^{\circ}$ & $\beta=45^{\circ}$ & $\beta=60^{\circ}$ & - & - \\
\hline Trailing edge curvature (TC) & $r=5 \mathrm{~mm}$ & $r=10 \mathrm{~mm}$ & $r=15 \mathrm{~mm}$ & $r=20 \mathrm{~mm}$ & - \\
\hline Cover plate $(\mathrm{CP})$ & $\alpha=0^{\circ}$ & $\alpha=15^{\circ}$ & $\alpha=30^{\circ}$ & $\alpha=45^{\circ}$ & - \\
\hline Jet blowing (JB) & T1 only & $\mathrm{T} 1$ and $\mathrm{T} 2$ & L1 only & $\mathrm{L} 1$ and $\mathrm{L} 2$ & All active \\
\hline
\end{tabular}

\section{Results}

The results for the baseline case will be presented in the first section of this part and then the results for the control cases will be shown in detail.

3.1. Results of the Baseline Computations. Table 4 shows the results for the frequency of the first mode of oscillations. The prediction from the Rossiter formulation for this frequency is $1109 \mathrm{~Hz}$. As seen in Table 3, grids 2, 3, 4, and 5 have similar results in terms of frequencies. The first and the last grids deviate.

The fourth grid was selected for further computations, both based on the flow structures and frequencies. The prediction for the second mode frequency is $2218 \mathrm{~Hz}$. The second mode frequency is predicted as $2167 \mathrm{~Hz}$ with computations with an error of $2.3 \%$ calculated based on Rossiter's theoretical formula. The experimental data of Kaufman et al. [17] shows the sound pressure levels at six locations on cavity floor. Table 5 shows the experimental results, their location, and computational predictions for the same location with the selected grid.

Pressure fluctuations for the baseline case together with the streamlines are shown in Figure 5. The formation of vortices due to shear layer reattachment, pressure fluctuations inside the cavity during the whole feedback cycle, bending of the shear layer causing mass to leave the cavity, and the formation of high pressure zones especially at the trailing edge wall of the cavity are shown in the figure for one Rossiter period. The pressures are much higher at the trailing edge wall than the leading edge wall. A circulation region fills the cavity. Shocks and vortices interact which causes pressure fluctuations. Figure 6 shows the density contours for the baseline case which clearly demonstrate the supersonic nature of the flow. The feedback mechanism inside the cavity is also observed in density contours as in the pressure contours. Kelvin Helmholtz instability of the shear layer can also be detected in the density contours. The temperature contours for several time steps are presented in Figure 7 . The $x$-velocity contours and streamlines are shown in Figure 8. They also demonstrate the vortex region that is formed inside the cavity clearly.

3.2. Results for Controlled Computations. Firstly, the results of different techniques will be presented and then, the results comparing the most effective case of each control method will be shown. The comparisons are made in terms of pressure values at the control point on the trailing edge wall, which is the location where the pressure fluctuations are expected to be maximum and Fast Fourier Transform (FFT) applied to this pressure distribution, and sound pressure levels for the cavity walls (front, back, and bottom walls). The location of the control point on the trailing edge wall is 0.6 cavity depth away from the bottom and 0.4 cavity depth away from the trailing edge of the cavity. Power spectra at several locations on the cavity floor are also presented both for the baseline case and for controlled simulations for comparison purposes. Pressure, density, streamwise velocity contours, and streamlines for various time steps are used for comparison, as well.

Figure 9 shows the pressure fluctuations and power spectrum for the control point for the baseline case with no flow control for comparison purposes. The pressure is nondimensionalized by free stream pressure and the time is nondimensionalized by the Rossiter period which is 857 time steps corresponding to 857 microseconds. As seen from the figure, the pressure oscillations are periodic. The dominant frequency $(2167 \mathrm{~Hz})$ corresponds to two Rossiter frequencies 

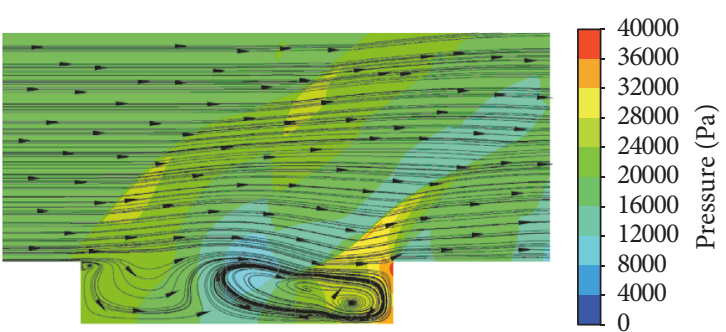

(a)

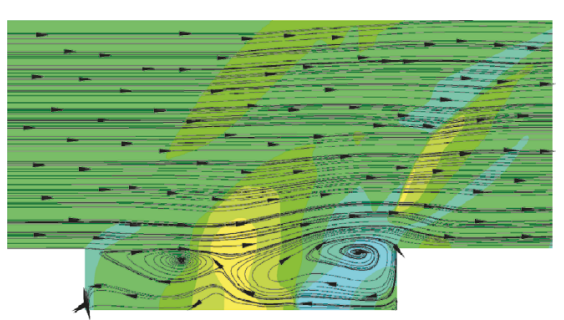

(c)

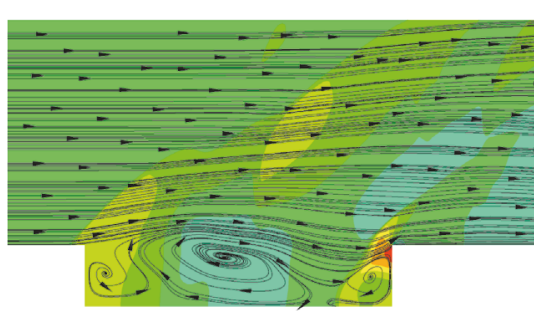

(e)

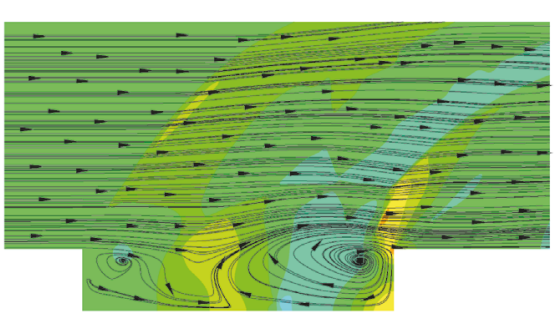

(g)

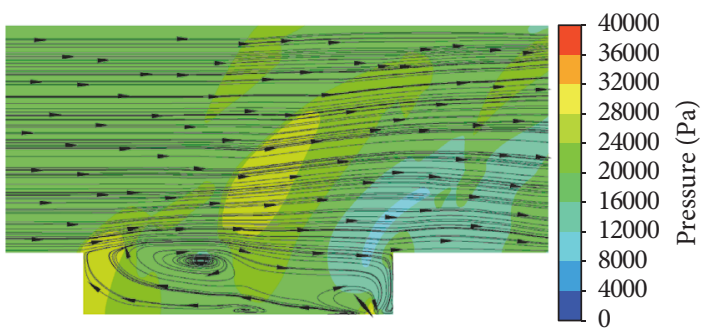

(i)

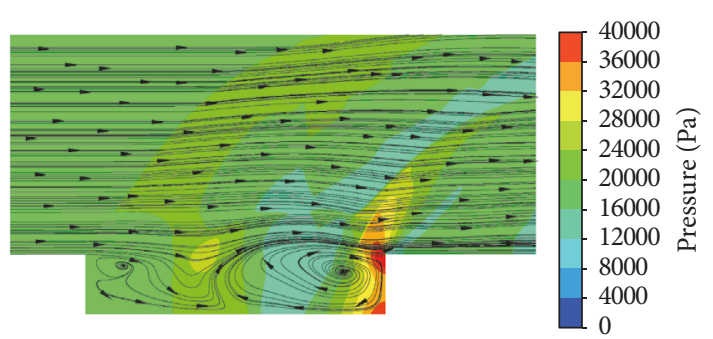

(b)

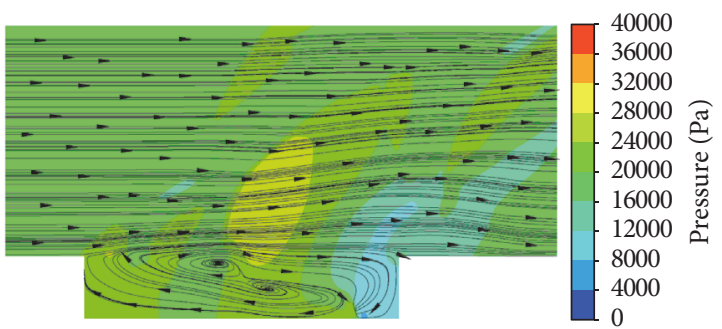

(d)

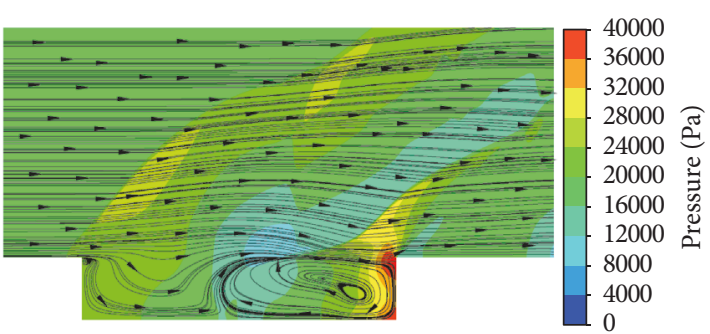

(f)

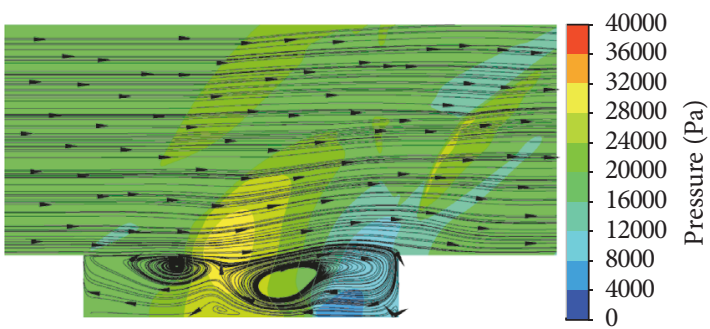

(h)

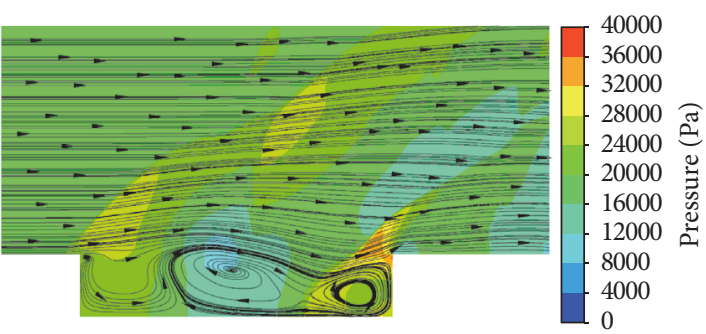

(j)

Figure 5: Pressure fluctuations of the whole domain for the baseline case (a) $t / t_{R}=0$, (b) $t / t_{R}=0.11$, (c) $t / t_{R}=0.22$, (d) $t / t_{R}=0.33$, (e) $t / t_{R}=0.44$, (f) $t / t_{R}=0.55$, (g) $t / t_{R}=0.66$, (h) $t / t_{R}=0.77$, (i) $t / t_{R}=0.88$, and (j) $t / t_{R}=1$.

which is predicted as $2218 \mathrm{~Hz}$ by Rossiter's formula. The difference between the computational results and Rossiter's formula is $2.3 \%$.

Figure 10 shows the pressure distribution and power spectra at three different locations on the cavity floor, where nondimensional streamwise distance from the leading edge $(x / L)$ is $0,0.4$, and 0.8 . As seen in this figure, the pressure distribution and power spectrum at the cavity floor show a similar trend with the control point.

3.3. Cover Plate. A cover plate shown in Figure 11 is applied to the cavity as a passive control method. The slope of the plate is 

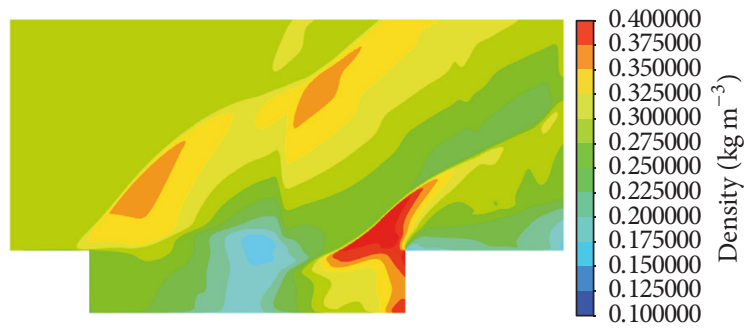

(a)
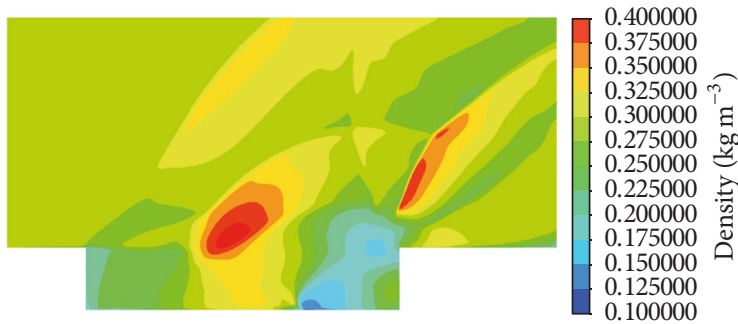

(c)
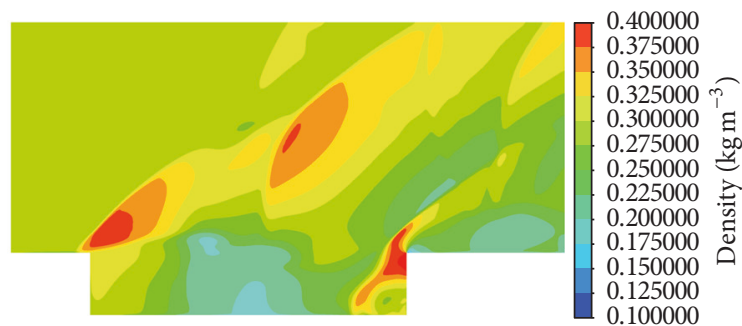

(e)

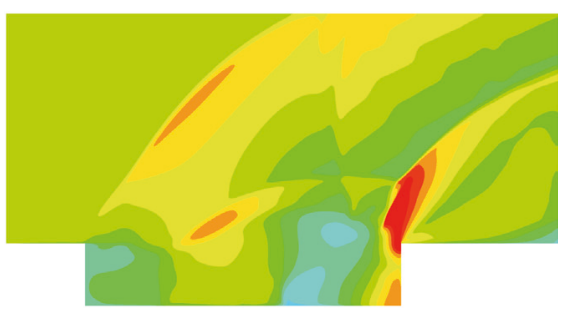

(g)

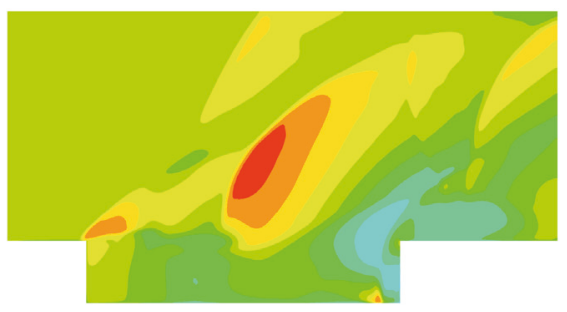

(i)

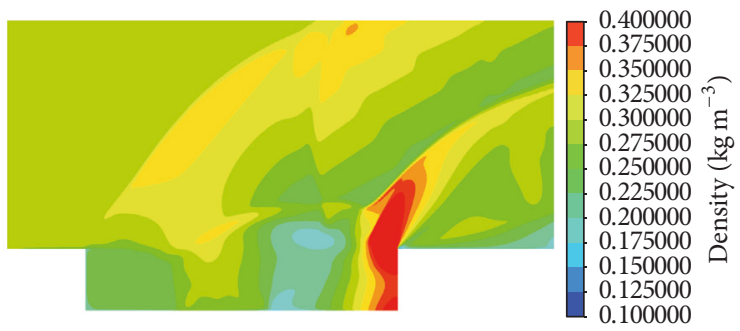

(b)

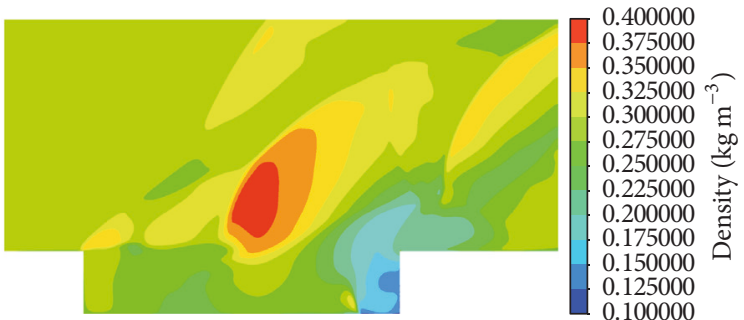

(d)

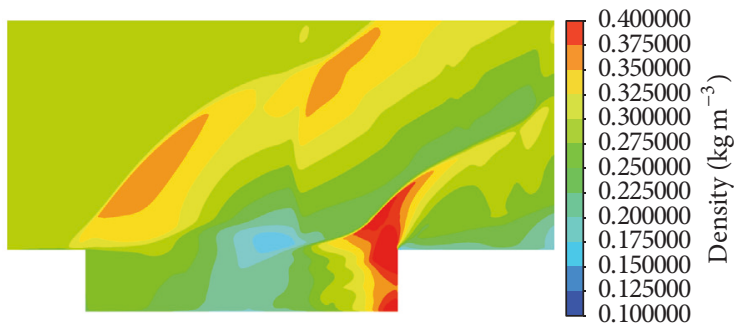

(f)
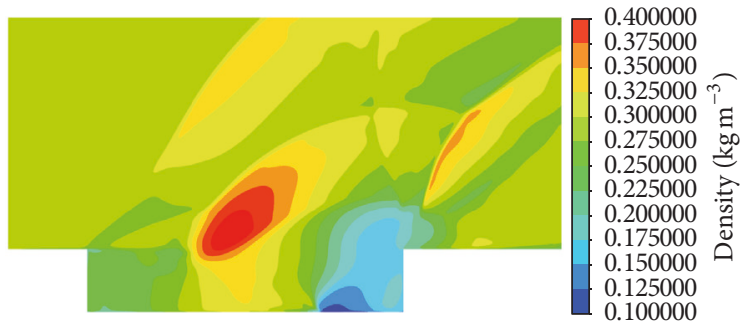

(h)
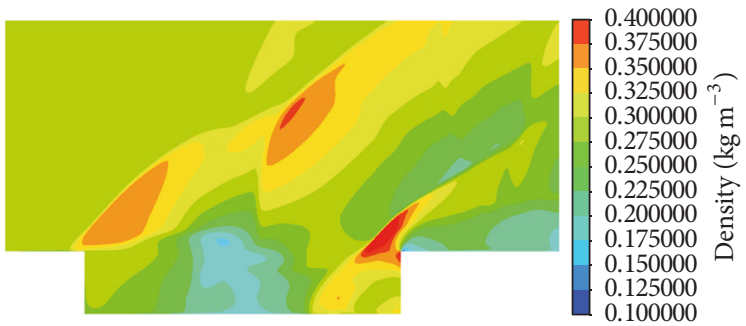

(j)

Figure 6: Density contours for the baseline case (a) $t / t_{R}=0$, (b) $t / t_{R}=0.11$, (c) $t / t_{R}=0.22$, (d) $t / t_{R}=0.33$, (e) $t / t_{R}=0.44$, (f) $t / t_{R}=0.55$, (g) $t / t_{R}=0.66$, (h) $t / t_{R}=0.77$, (i) $t / t_{R}=0.88$, and (j) $t / t_{R}=1$.

0 (CP1), 15 (CP2), 30 (CP3), and 45 (CP4) degrees as shown in detail in Table 3. The plate with no slope is shown in Figure 11 for demonstration purposes. The length of the plate is $25 \%$ of the cavity length and the thickness is $2 \mathrm{~mm}$.
Figure 12(a) shows the pressure distribution at the control point for all cover plate control cases. Figure 12(b) shows the FFT analysis of this pressure distribution to observe the dominant frequencies. The FFT analysis of all cover plate control 

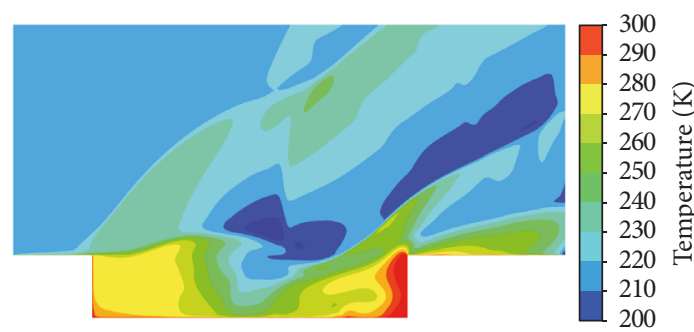

(a)

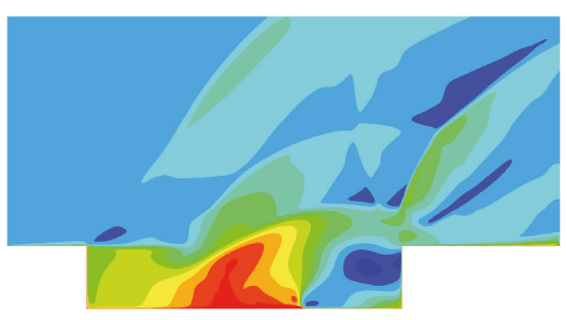

(c)

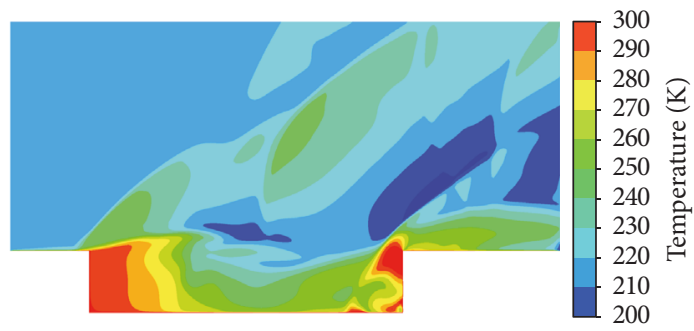

(e)

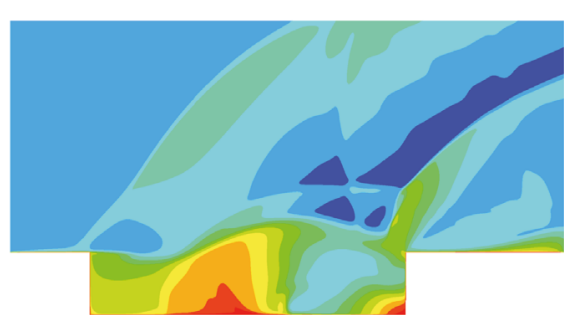

(g)

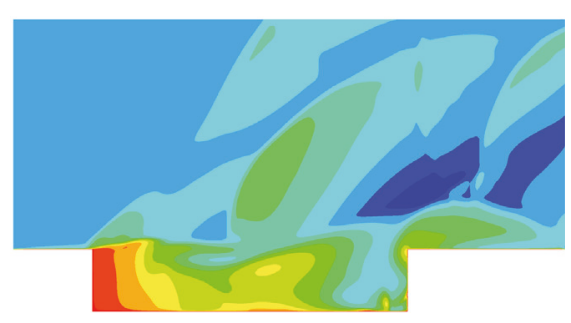

(i)

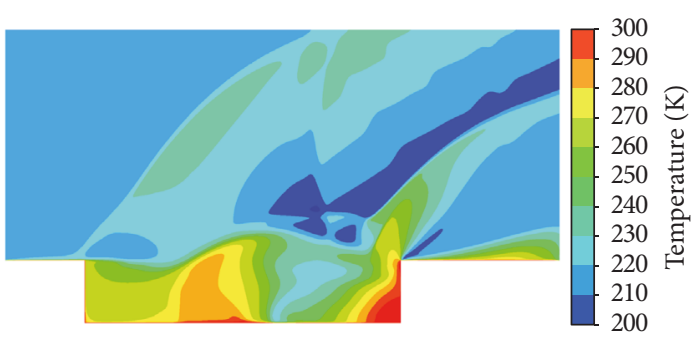

(b)

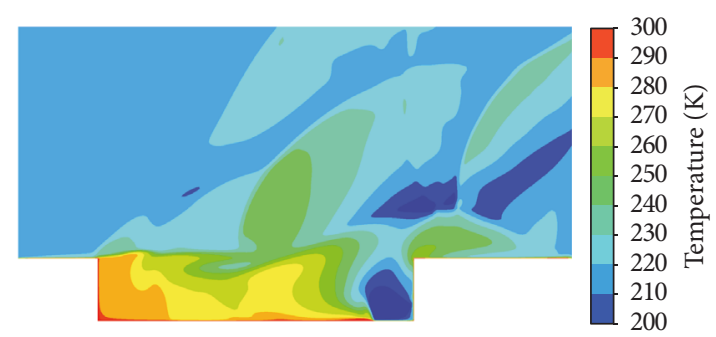

(d)

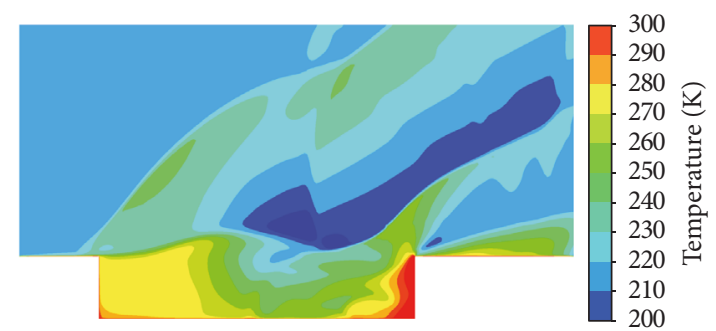

(f)

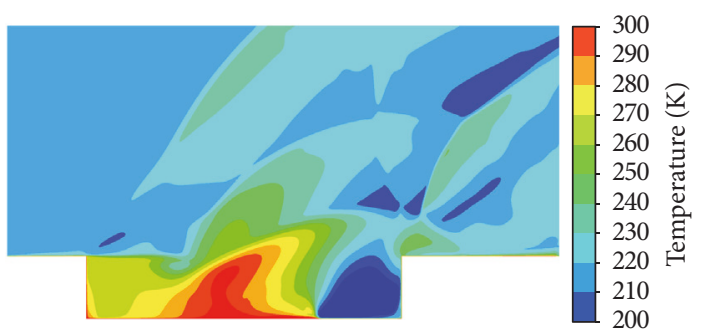

(h)

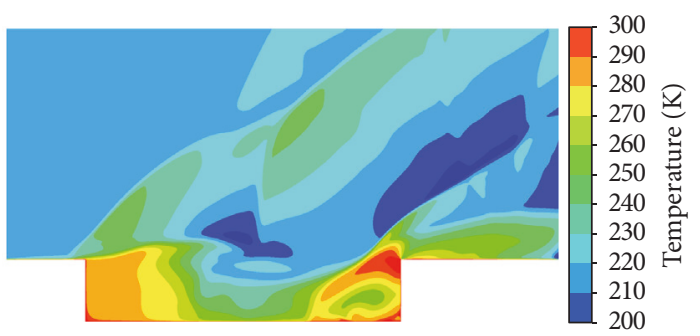

(j)

Figure 7: Temperature contours for the baseline case (a) $t / t_{R}=0$, (b) $t / t_{R}=0.11$, (c) $t / t_{R}=0.22$, (d) $t / t_{R}=0.33$, (e) $t / t_{R}=0.44$, (f) $t / t_{R}=0.55$, (g) $t / t_{R}=0.66$, (h) $t / t_{R}=0.77$, (i) $t / t_{R}=0.88$, and (j) $t / t_{R}=1$.

cases provide similar results; therefore only the FFT results of the CP4 case (45 degrees) are shown for demonstration purposes. Figure 9 clearly shows a dominant frequency at $2167 \mathrm{~Hz}$ (corresponding to two Rossiter periods); however, the dominant frequency in Figure 12 is more than $3000 \mathrm{~Hz}$ which corresponds to approximately three Rossiter periods. The pressure distributions and dominant frequencies are altered with the use of cover plate. When the slope of the cover plate increases, the magnitude of pressure oscillations decreases, but the periodicity of the flow remains. However, 


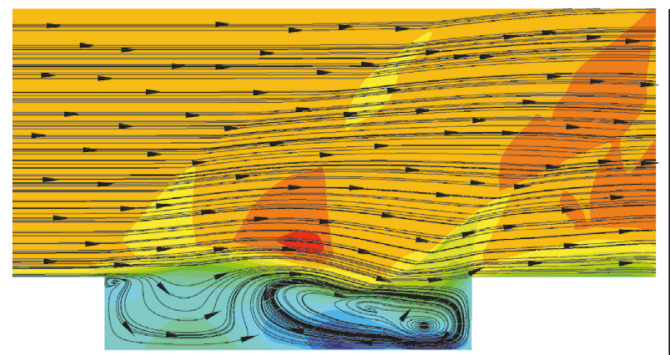

(a)

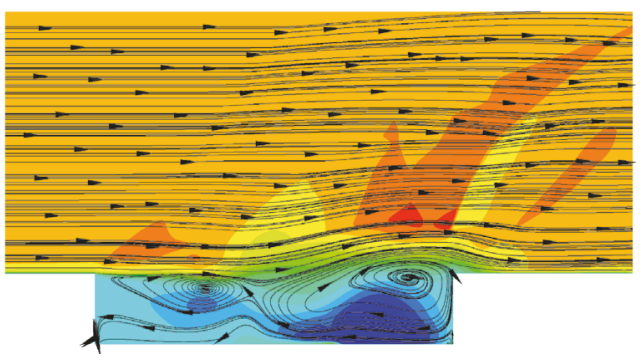

(c)

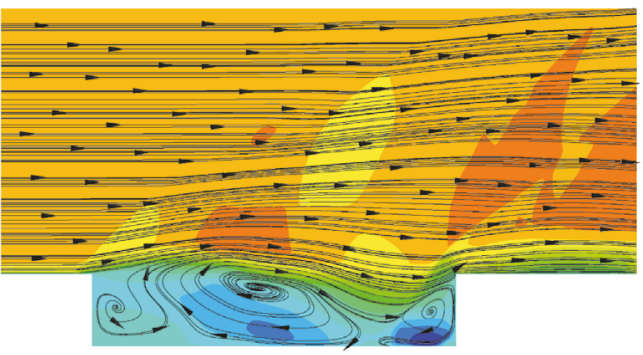

(e)

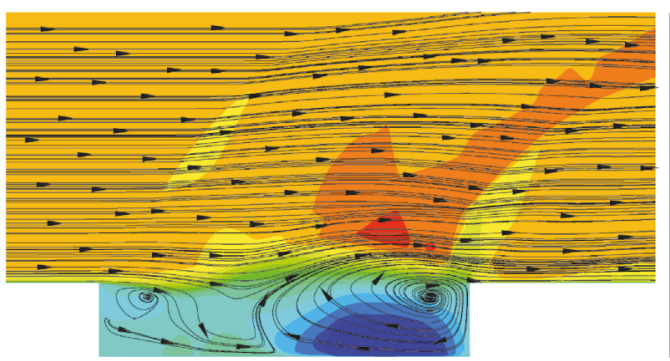

(g)

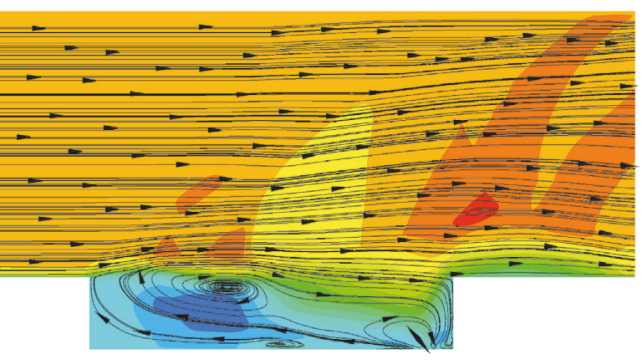

(i)
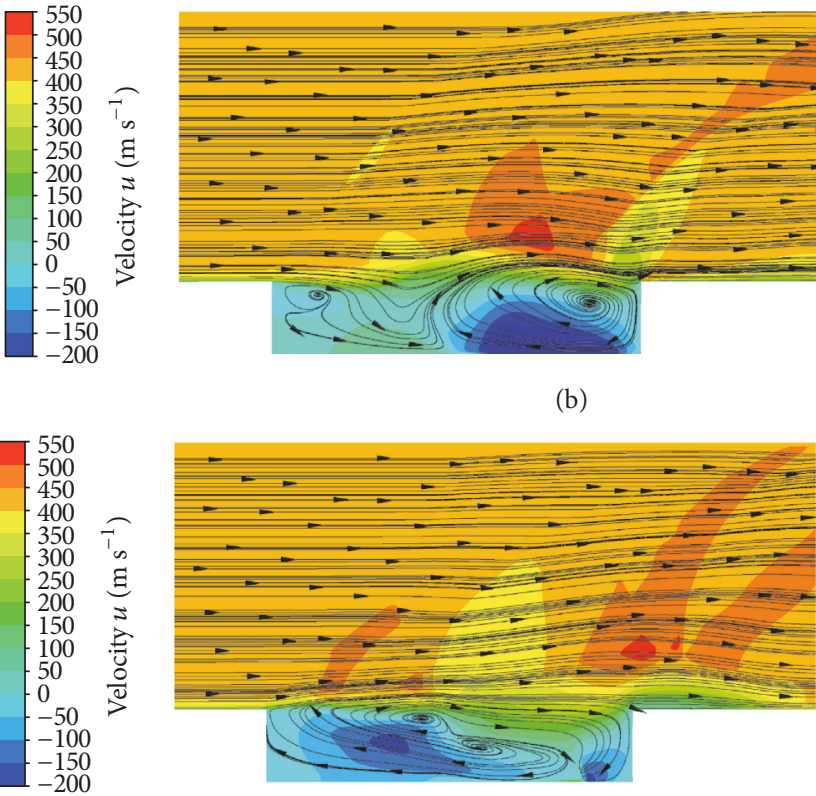

(d)
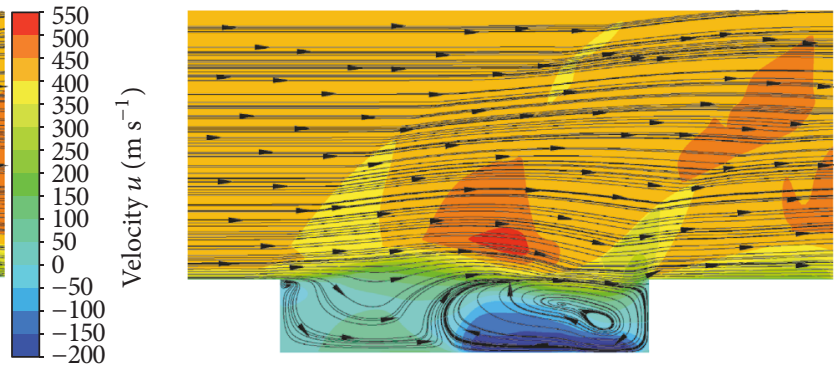

(f)

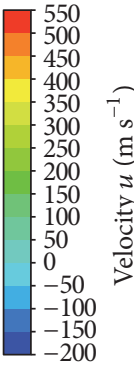

$-150$
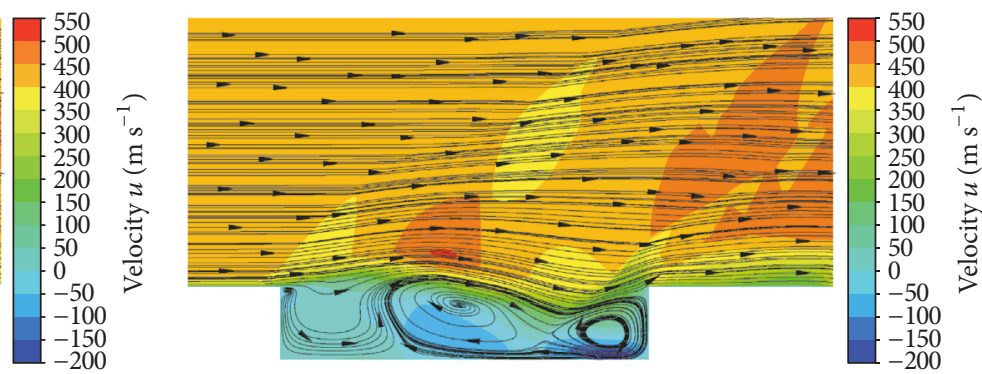

(j)

FiguRE 8: Contours of velocity in streamwise direction and streamlines for the baseline case (a) $t / t_{R}=0$, (b) $t / t_{R}=0.11$, (c) $t / t_{R}=0.22$, (d) $t / t_{R}=0.33$, (e) $t / t_{R}=0.44$, (f) $t / t_{R}=0.55$, (g) $t / t_{R}=0.66$, (h) $t / t_{R}=0.77$, (i) $t / t_{R}=0.88$, and (j) $t / t_{R}=1$. 


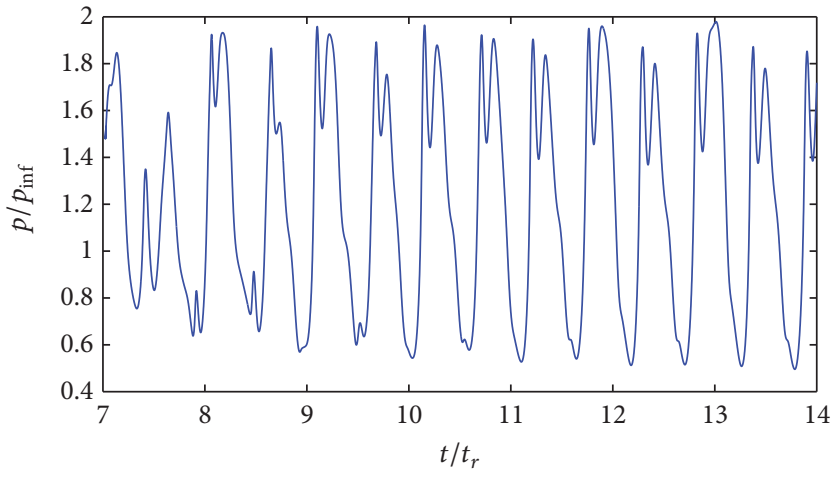

(a)

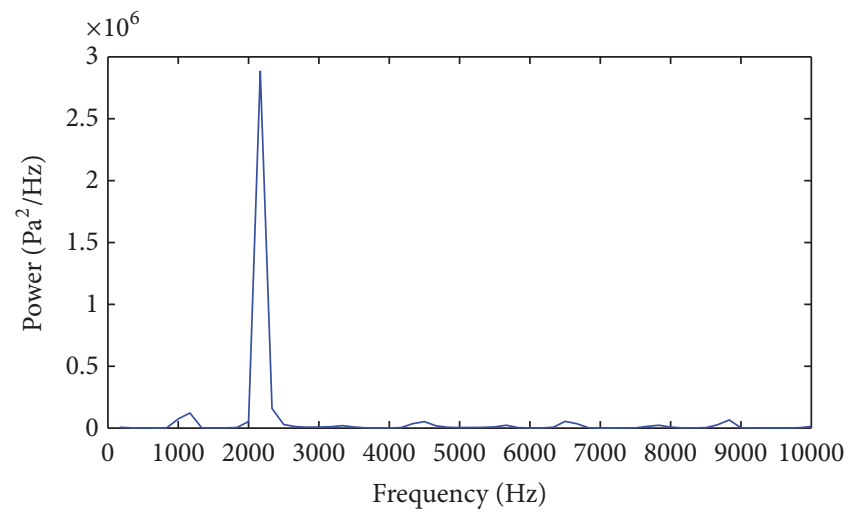

(b)

Figure 9: The results for the control point for baseline case. (a) Pressure distribution. (b) FFT analysis of the pressure data.
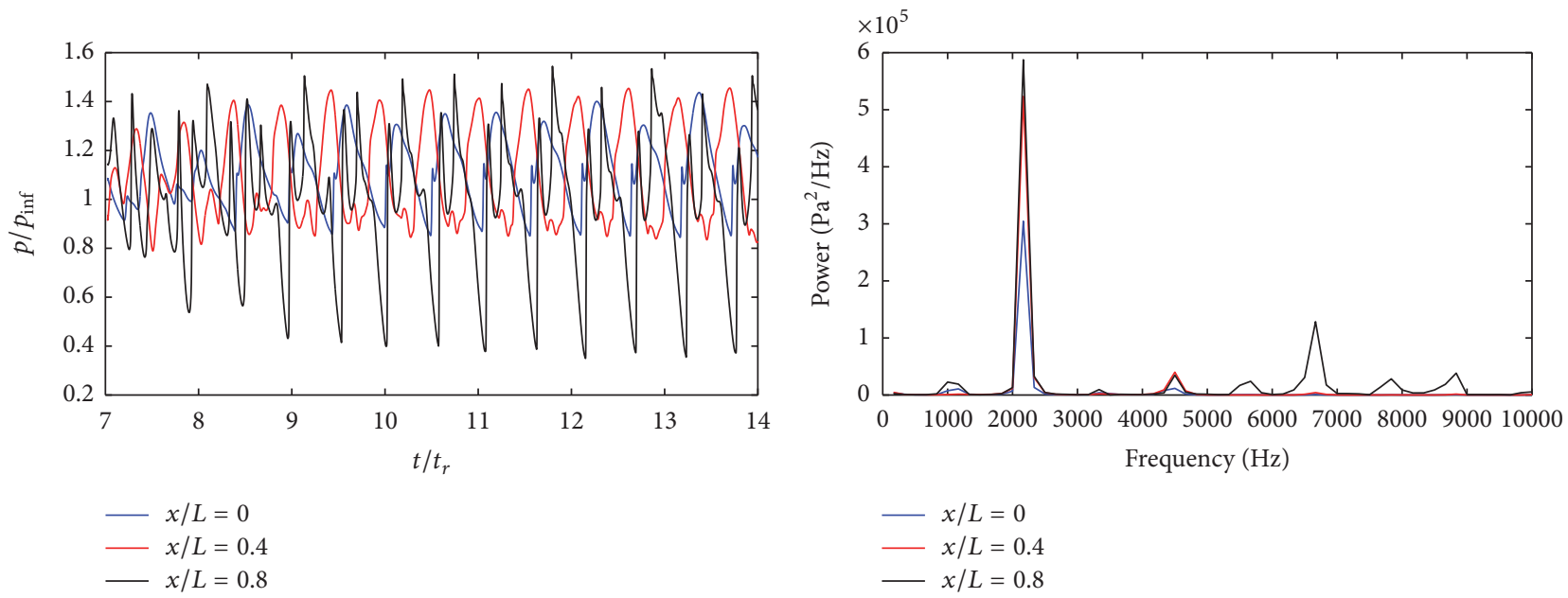

(a)

(b)

Figure 10: The results for cavity floor. (a) Pressure distribution. (b) Power spectrum for the baseline case.

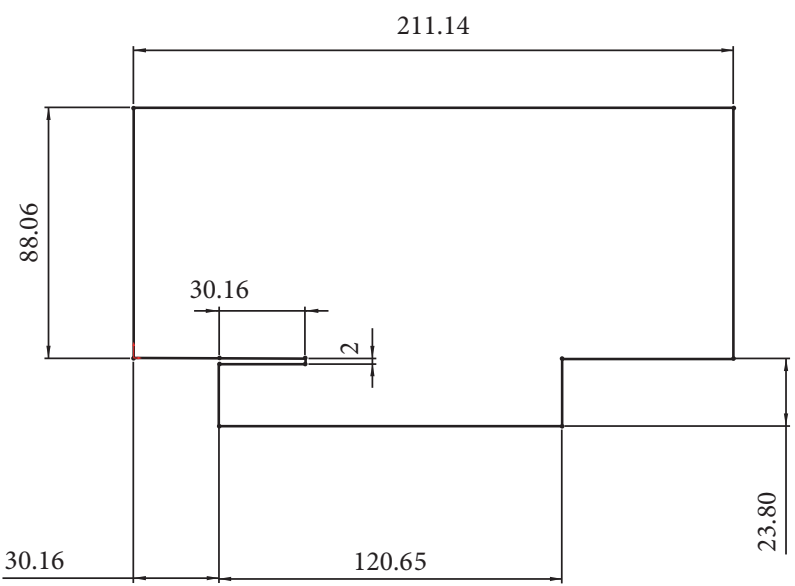

FIGURE 11: Cover plate geometry. 


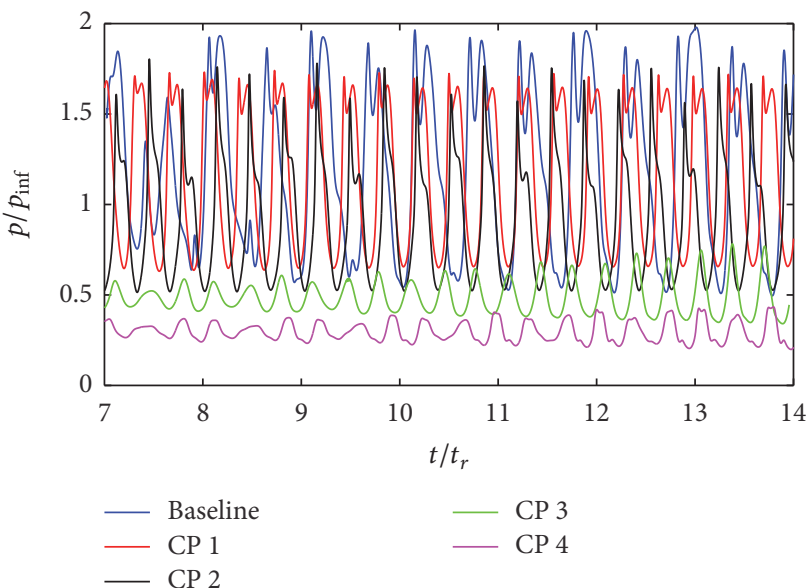

(a)

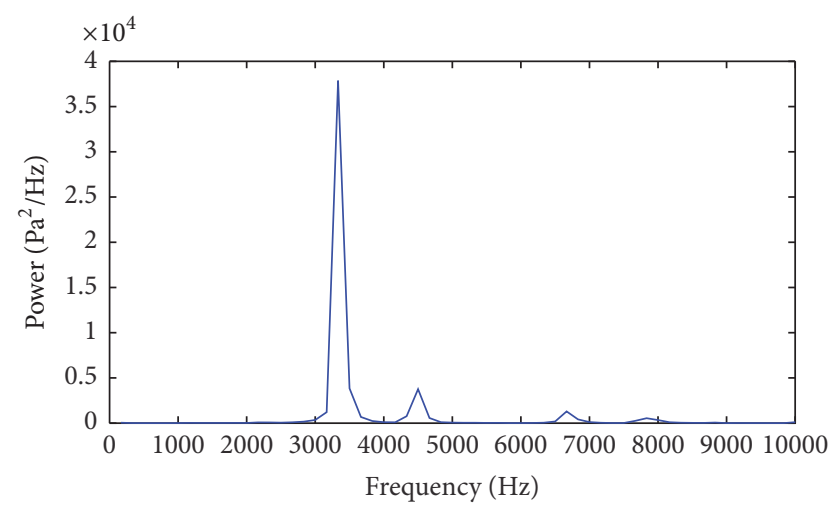

(b)

FIGURE 12: The results for the control point. (a) Pressure distribution for the baseline case and the cases with cover plate. (b) FFT analysis for the pressure data obtained from the control point (CP4).

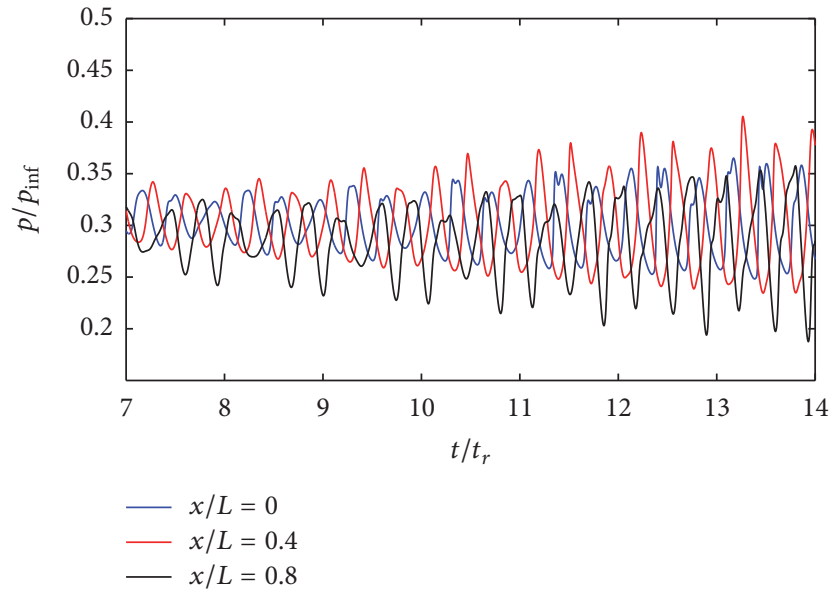

(a)

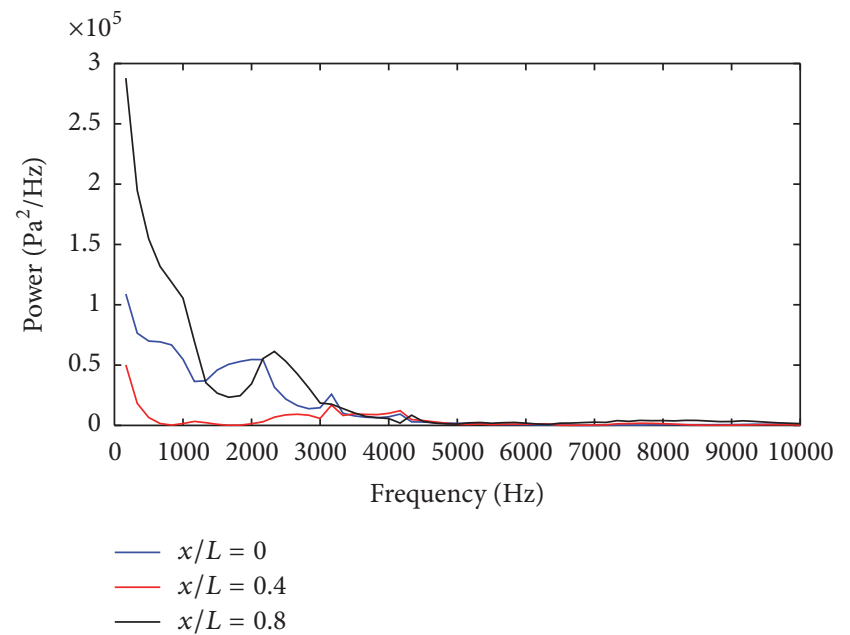

(b)

FIGURE 13: The results for cavity floor $(x / L=0,0.4$, and 0.8). (a) Pressure distribution. (b) Power spectrum (CP4).

there is a clear shift in periods, as also shown in the FFT graph. Similar trend is shown for several locations at the cavity floor $(x / L=0,0.4$, and 0.8$)$ in terms of pressure history and power spectra in Figure 13, for CP4 case.

Figure 14 shows the sound pressure levels in terms of decibels for the bottom wall, leading edge wall, and trailing edge wall when cover plates are used. The trend in pressure distribution at the control point is also observed in the sound pressure level (SPL) distributions. Increasing the cover plate slope decreases the magnitude of the pressure oscillations; therefore, it also decreases the SPL values for the cavity walls.

Figures 15 and 16 show the density contours and $x$ velocity contours with streamlines, respectively, for $\mathrm{CP} 4$ configuration which is the most effective configuration of control cases with cover plate. The cover plate acts as a barrier for the incoming flow and breaks the periodic feedback structure inside the cavity. The shear layer is distorted and the flows before and after the cover plate act like two different zones. The distortion in the shear layer gives more space to the vortex inside the cavity. This lets the flow have a lower streamwise velocity inside the cavity and the velocity increase takes place further away from the inside, as observed in Figure 16 that shows the streamlines and $x$-velocity contours. The densities are very high before the cover plate. However, they are much lower at the region behind the plate. The reason is most probably the change in the feedback mechanism due to the plate. When Figures 5, 6, and 8 for the baseline case are examined, it is observed that the periodic nature of the flow is due to the formation and impingement of the vortices inside the cavity to the trailing edge wall. However, with the cover plate, this is not the case. This loop is broken and the vortices do not collapse on the trailing edge wall; instead more flow 


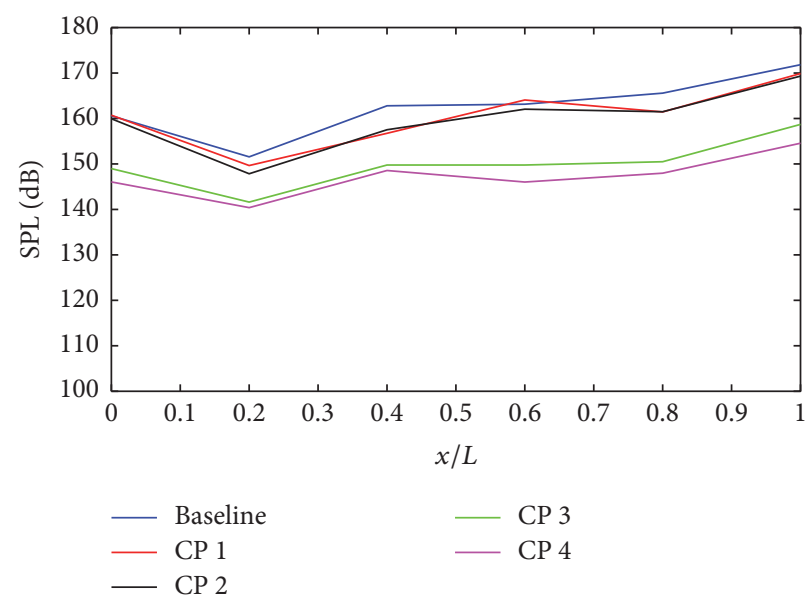

(a)

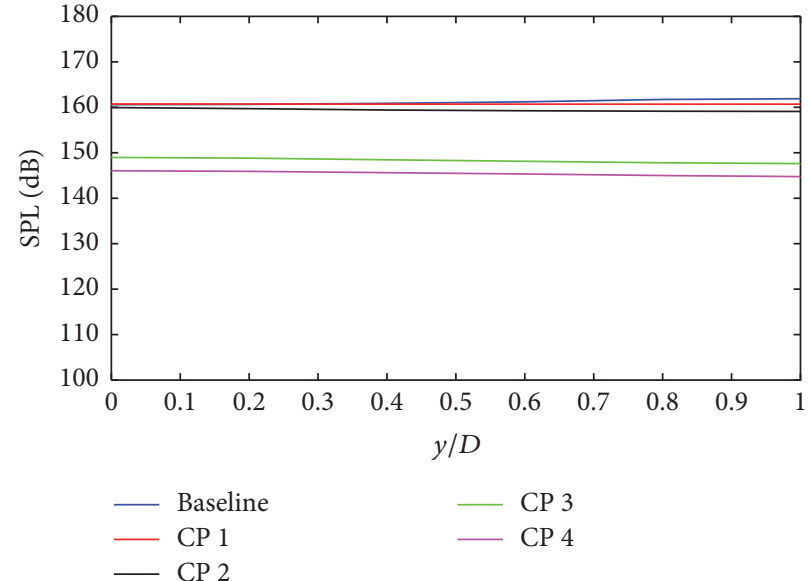

(b)

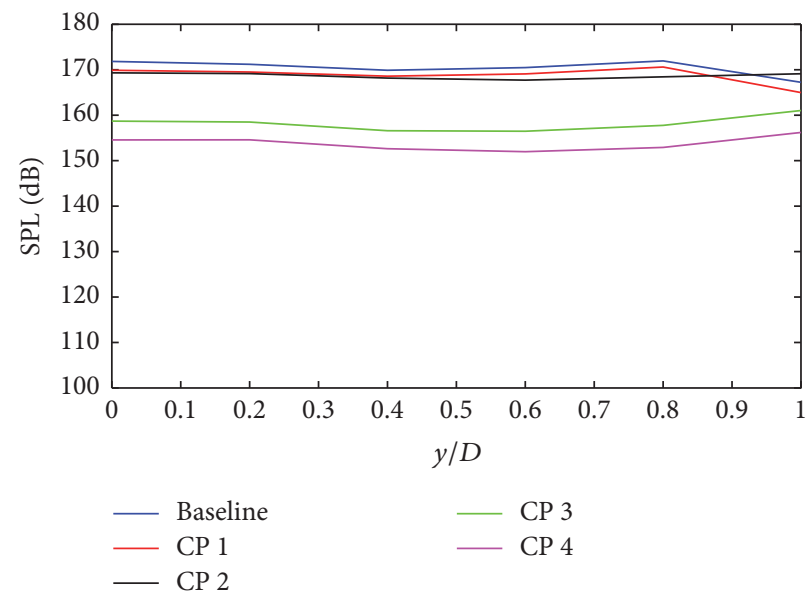

(c)

FIGURE 14: Sound pressure levels for cavity walls for cover plate cases. (a) Bottom wall. (b) Leading edge wall. (c) Trailing edge wall.

TABLE 4: Frequency predictions with different grid resolutions.

\begin{tabular}{lcc}
\hline Grid & Rossiter frequency (1st mode) & \% difference with Rossiter frequency formulation \\
\hline 1 & 1230 & 11 \\
2 & 1195 & 7.7 \\
3 & 1037 & 6.5 \\
4 & 1166 & 5.22 \\
5 & 1050 & 5.3 \\
6 & 1010 & 9 \\
\hline
\end{tabular}

TABLE 5: Comparison of computations and experiments for cavity floor sound pressure levels.

\begin{tabular}{lccr}
\hline Cavity floor location $(x / L)$ & SPL (experiment $[17])(\mathrm{dB})$ & SPL (computation) & \% difference \\
\hline 0 & 152 & 160 & 5.3 \\
0.2 & 147 & 150 & 2.0 \\
0.4 & 153 & 161 & 5.2 \\
0.6 & 154 & 160 & 3.9 \\
0.8 & 155 & 160 & 3.2 \\
1.0 & 162 & 168 & 3.7 \\
\hline
\end{tabular}



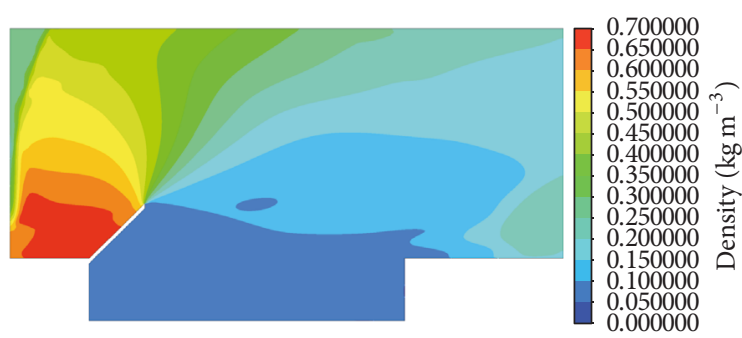

(a)
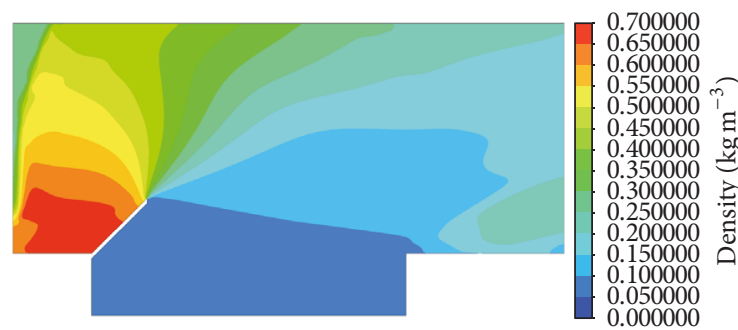

(c)
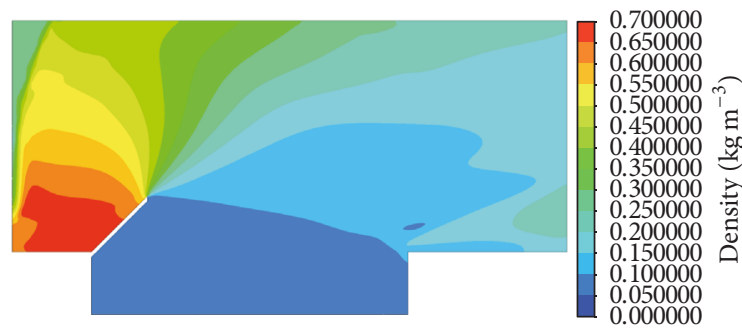

(e)

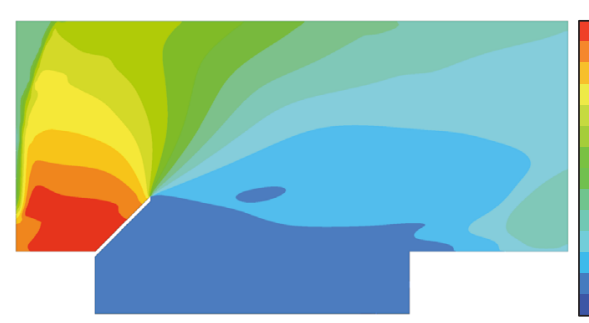

(g)

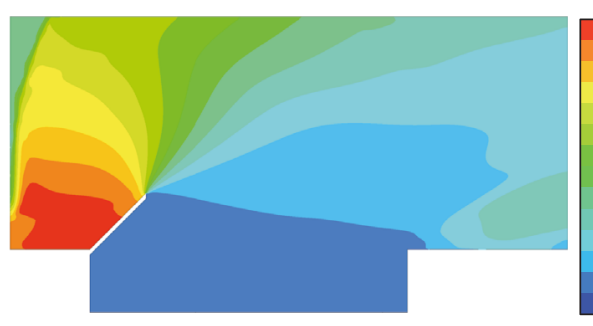

(i)

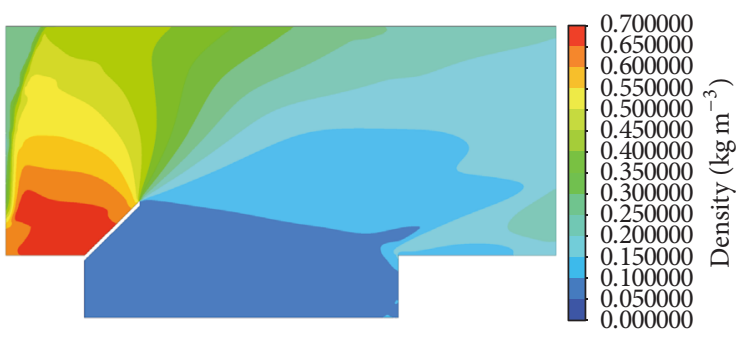

(b)

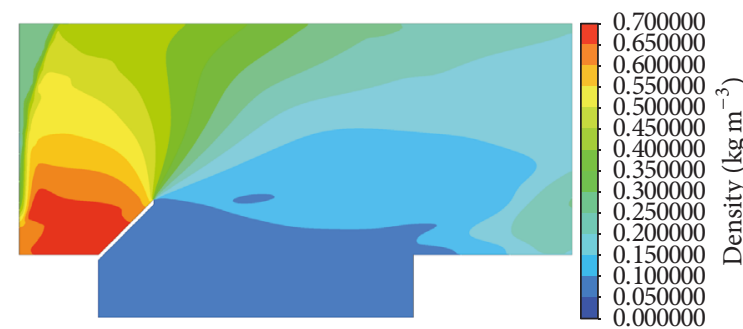

(d)

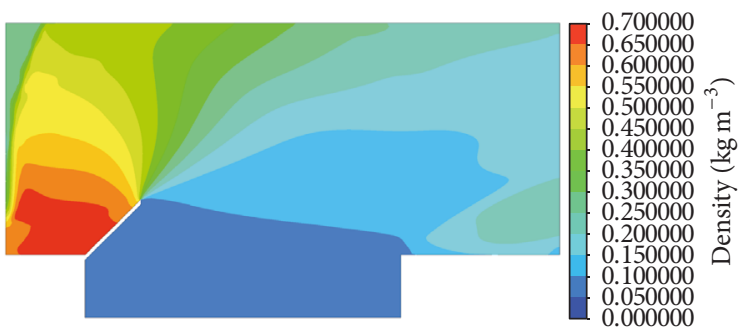

(f)

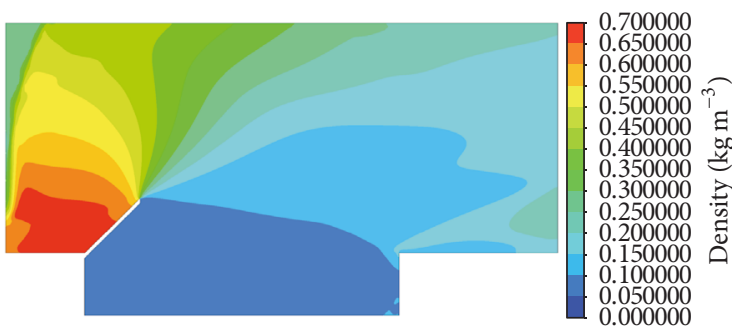

(h)

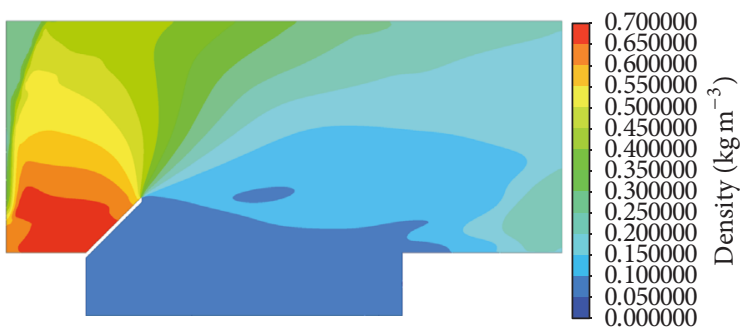

(j)

Figure 15: Density contours for CP4 case (a) $t / t_{R}=0$, (b) $t / t_{R}=0.11$, (c) $t / t_{R}=0.22$, (d) $t / t_{R}=0.33$, (e) $t / t_{R}=0.44$, (f) $t / t_{R}=0.55$, (g) $t / t_{R}=0.66$, (h) $t / t_{R}=0.77$, (i) $t / t_{R}=0.88$, and (j) $t / t_{R}=1$.

goes smoothly over the cavity. As observed from all the graphs shown, CP4 which has a slope of $45^{\circ}$ is the most effective of all. As the angle increases, the blockage of the entrance to cavity decreases. Also, increased angles provide more space to the vortex inside the cavity and more space for shear layer deformation, which makes it more convenient to use higher slopes. However, if the slope is increased more than necessary, it can block the incoming flow directly and may act as a shock; therefore, it is reasonable to have the best results in terms of pressure fluctuations with CP4. 


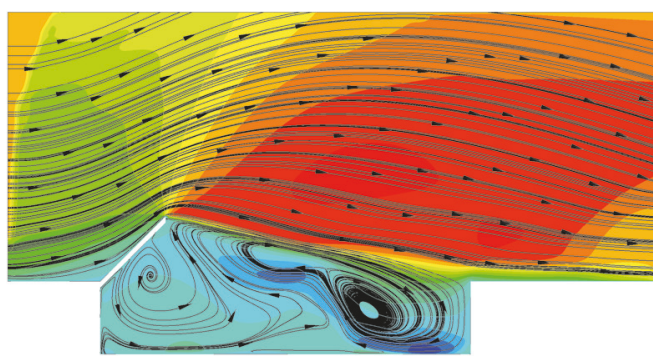

(a)

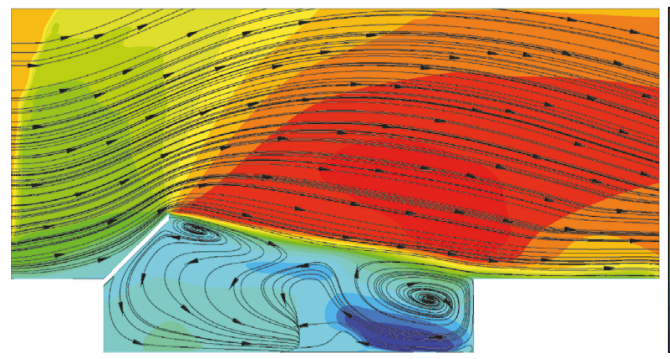

(c)

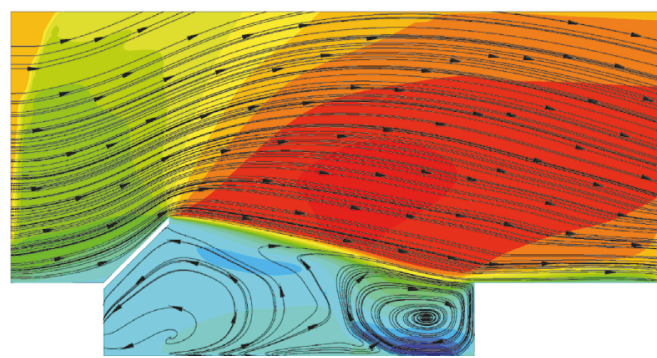

(e)

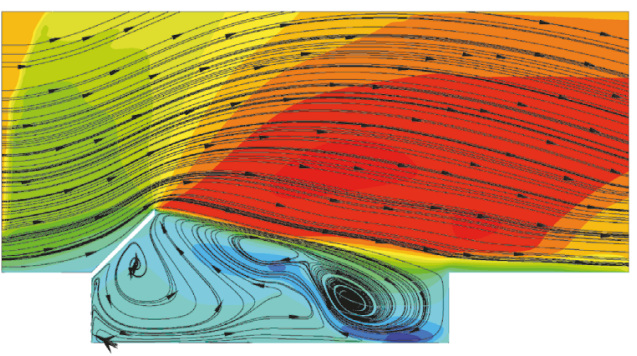

(g)

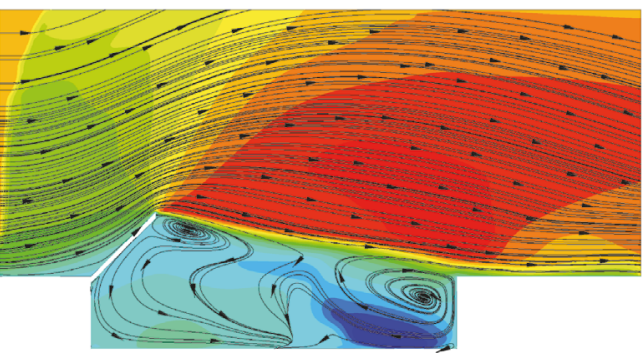

(i)
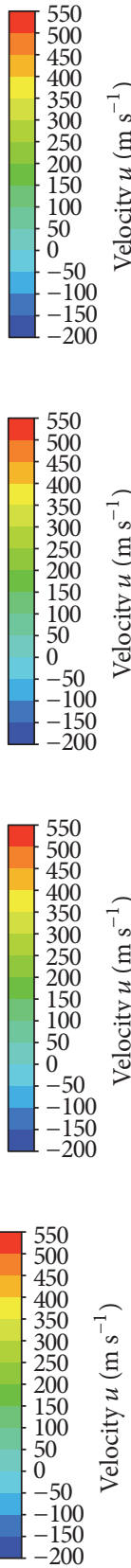

$-150$

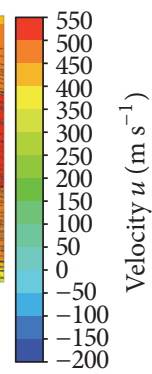

$-150$
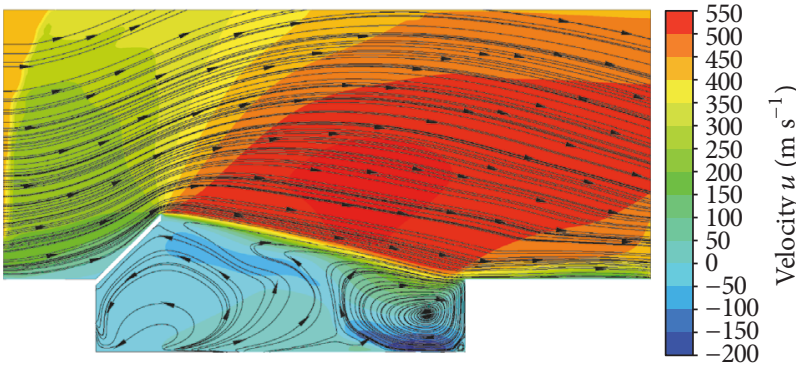

(b)
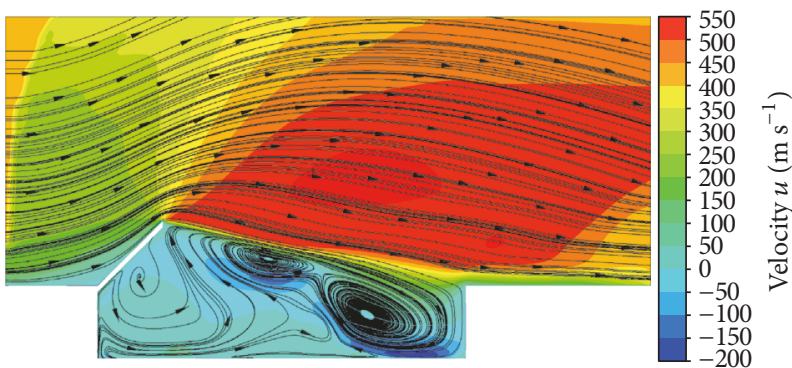

(d)
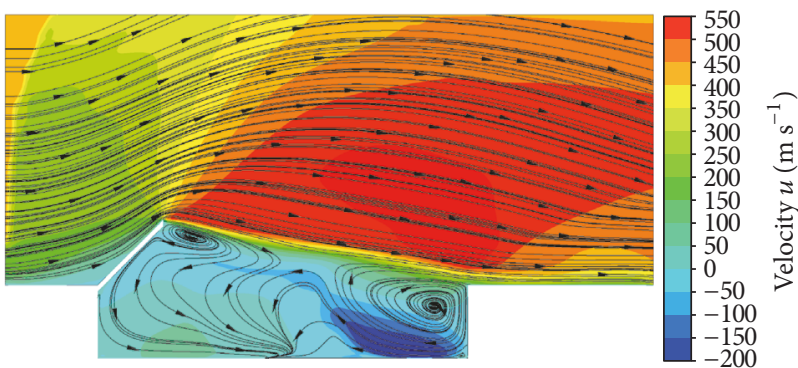

(f)
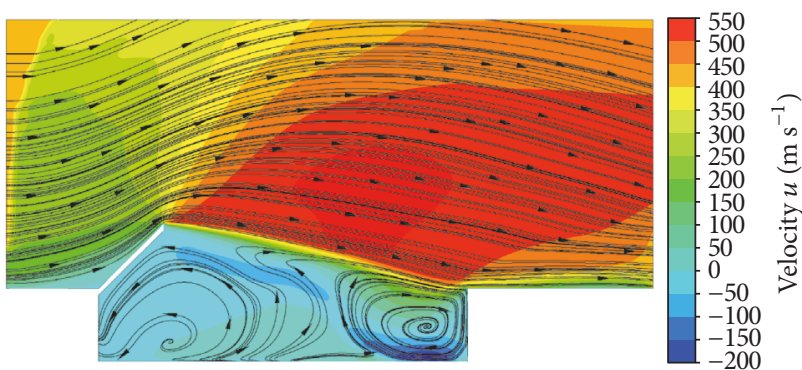

(h)
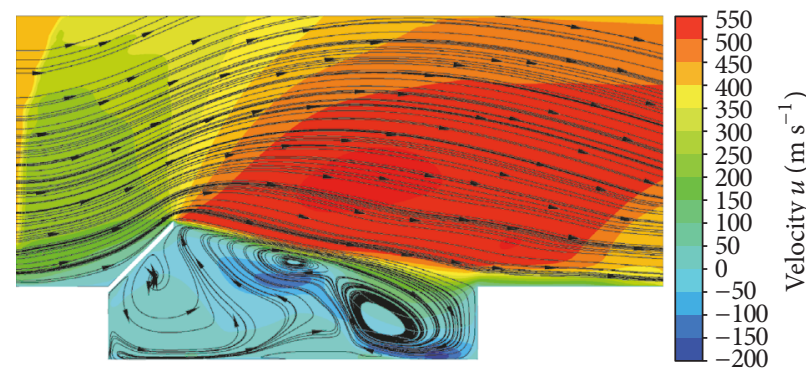

(j)

FIGURE 16: $X$-velocity contours and streamlines for CP4 case (a) $t / t_{R}=0$, (b) $t / t_{R}=0.11$, (c) $t / t_{R}=0.22$, (d) $t / t_{R}=0.33$, (e) $t / t_{R}=0.44$, (f) $t / t_{R}=0.55$, (g) $t / t_{R}=0.66$, (h) $t / t_{R}=0.77$, (i) $t / t_{R}=0.88$, and (j) $t / t_{R}=1$. 


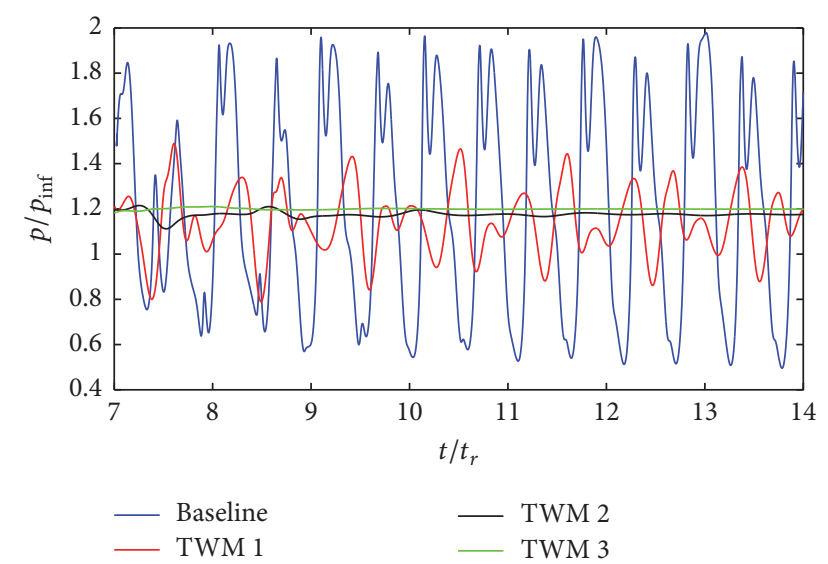

Figure 17: Pressure distribution at the control point with trailing edge wall modifications.

3.4. Trailing Edge Wall Modifications. The trailing edge wall of the cavity is modified by providing a slope of 30 (TWM1), 45 (TWM2), and 60 (TWM3) degrees. The details of the cases are shown in Table 3. Figure 17 shows the pressure distribution for the control point, whereas Figure 18 shows the SPL distribution for the bottom, leading edge, and trailing edge walls of the cavity. The pressure fluctuations (both magnitude and frequency) decrease drastically when the trailing edge wall of the cavity is modified.

Figure 19 shows the pressure fluctuations and power spectra at three different locations on cavity floor for the most effective trailing edge modification case: TWM3. Similar trend is observed as shown in previous figures: the pressure oscillations disappear on the cavity floor, as well.

Time dependent pressure fluctuations for the whole domain after trailing edge wall modification 3 (TWM3) which is the most effective of TWM cases are shown in Figure 20. The contours are provided with the same pressure levels for the baseline case (Figure 5) for comparison purposes. As clearly observed from the pressure contours and streamlines, the flow for the controlled case is not periodic; however, the baseline case shows the pressure oscillations and time dependent flow structure. Figures 21 and 22 show the density contours and $x$-velocity contours with streamlines for trailing edge wall modification 3 configuration. As seen in density, pressure, and $x$-velocity contours, as well as the streamlines, the flow structure is not periodic any more when the trailing edge wall is modified with a slope of 60 degrees. In the baseline case, a vortex develops inside the cavity and the movement of this vortex and interactions of it with the shear layer increase the pressure fluctuations inside. However, when the trailing edge wall is modified, the flow does not hit a straight trailing edge wall and go back to the leading edge, strengthening the vortex inside the cavity. On the contrary, more fluid goes over the sloped wall smoothly without causing pressures to change, leading the pressure oscillations to be alleviated. When the sound pressure levels are examined, it is observed that leading edge wall SPL values do not change drastically. However, since the trailing wall is smooth and has a slope (TWM 3), the SPL values on this wall decrease and the periodic nature of the flow disappears.
Figure 23 shows the density contours for baseline and all trailing edge wall modification cases (TWM 1, TWM 2, and TWM 3) for only one time value $\left(t / t_{R}=0.55\right)$. The baseline case clearly has the highest densities on its straight trailing edge wall. Introducing a slope at the trailing edge wall smoothens out the flow. Increasing the slope of the wall as much as possible lets more fluid go over the smooth wall and exit the cavity instead of helping to strengthen the vortex inside the cavity, which explains why TWM 3 with the highest slope is the most effective in alleviating pressure fluctuations.

3.5. Trailing Edge Curvature. As a third passive control method, the trailing edge wall radius is changed. The details are presented in Table 3. A radius of 5 (TC1), 10 (TC2), 15 (TC3), and $20 \mathrm{~mm}$ (TC4) is used to provide the curvature as shown in Figure 24 for $r=20 \mathrm{~mm}$, for demonstration purposes.

Figure 25 shows the pressure distribution for the control point. As seen clearly in the graph, the magnitude of the pressure oscillations decreases drastically when the trailing edge wall is provided with a curvature to let the air flow smoothly over the cavity. Increasing the radius of the curvature helps this smoothening; therefore, the magnitude of the oscillations further decreases with increased curvature.

A representative result of the FFT analysis is shown in Figure 26 for a radius of $15 \mathrm{~mm}$. The graph shows that the peaks are for the same frequencies as the baseline case (Figure 9); however, the magnitudes of the power accumulated at these frequencies differ shifting the dominant mode to higher frequencies.

The SPL values drop to 135 decibels for the bottom and front walls and 150 decibels for the trailing edge wall when curvature is provided to the trailing edge wall, which corresponds to $16 \%$ for the bottom and front walls and $11.5 \%$ for the trailing edge wall.

As seen both in pressure fluctuations and in power spectra, trailing edge curvature is not as effective as trailing edge wall modification by providing a slope. Periodic nature of the flow does not change; but the magnitude of the fluctuations decreases. Smoothening of the trailing edge wall basically provides a similar effect as introducing a trailing edge slope by letting the flow go over the cavity instead of hitting the edge and strengthening the vortex inside. Figure 27 shows the density contours for baseline and trailing edge curvature cases. As seen from the contours, increasing the radius smoothens the shear layer and incoming flow and lets more air flow over the cavity instead of hitting the wall, which then, in turn, helps to decrease the pressure oscillations caused by vortex-shear layer interactions.

3.6. Active Control: Jet Blowing. Jet blowing is utilized as an active control method. The location of the holes for jet blowing, and the dimensions of the geometry are shown in Figure 28. All the dimensions are given in millimeters. As shown in Table 3 , there are five different cases. The jet blowing velocity is taken as $10 \%$ of the free stream velocity for all cases. 


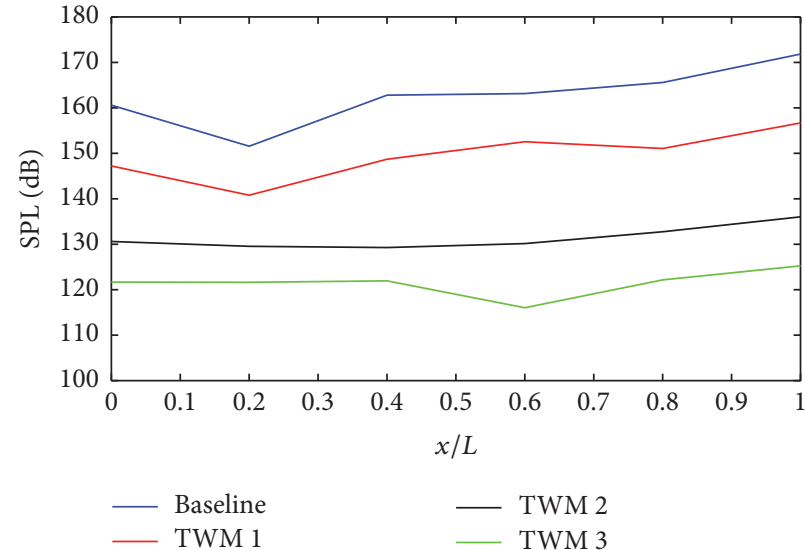

(a)

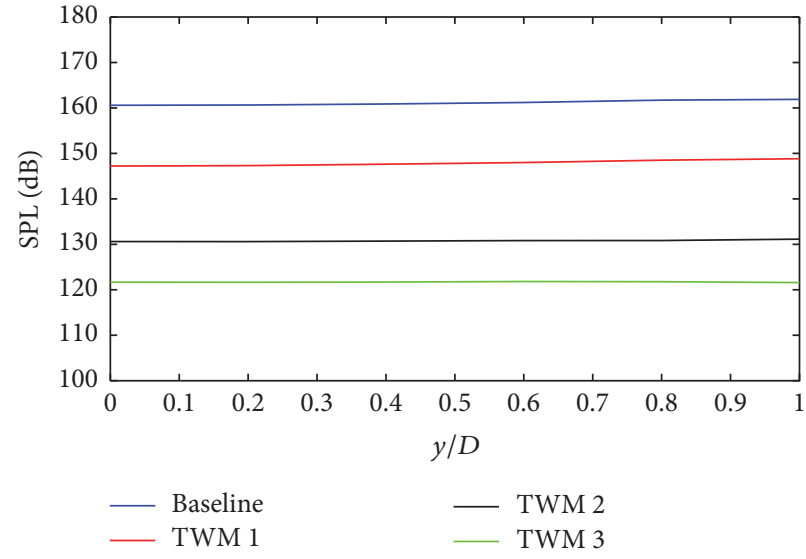

(b)

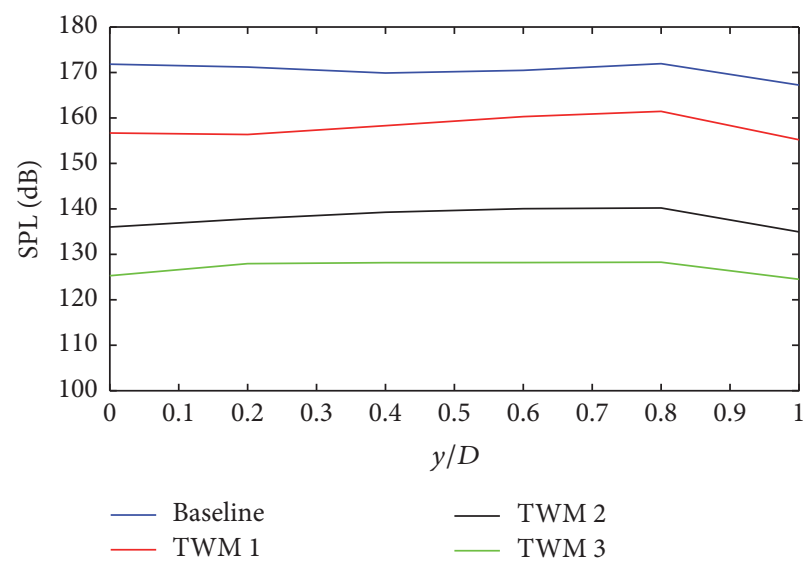

(c)

FIGURE 18: Sound pressure levels for cavity walls for TWM cases. (a) Bottom wall. (b) Leading edge wall. (c) Trailing edge wall.
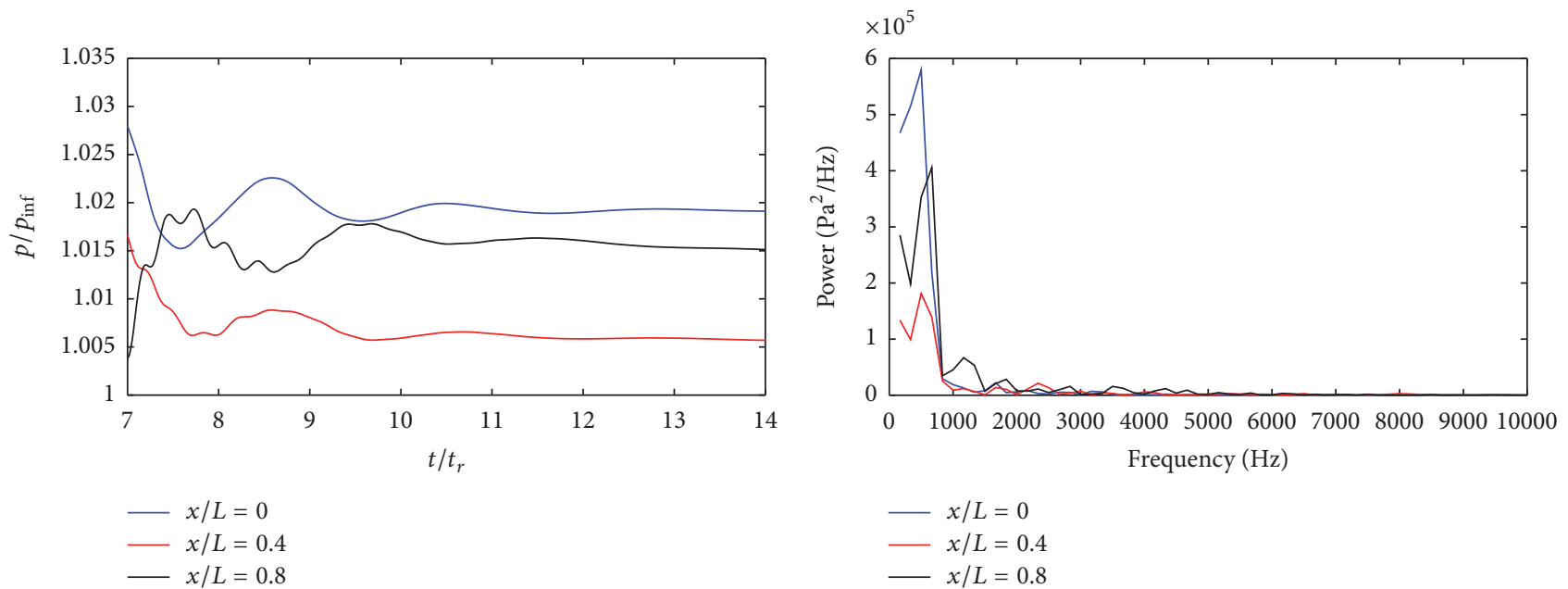

(a)

(b)

Figure 19: The results for cavity floor $(x / L=0,0.4$, and 0.8). (a) Pressure distribution. (b) Power spectrum (TWM 3). 


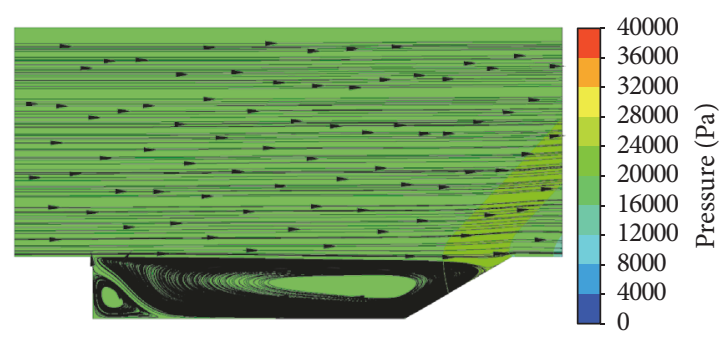

(a)

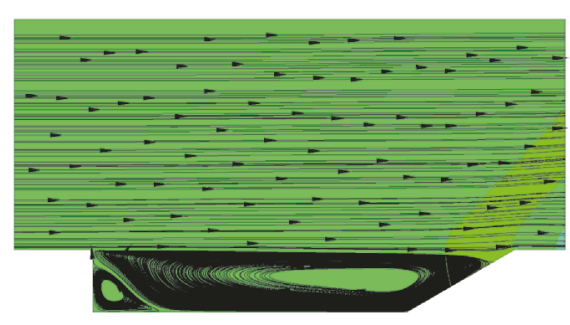

(c)

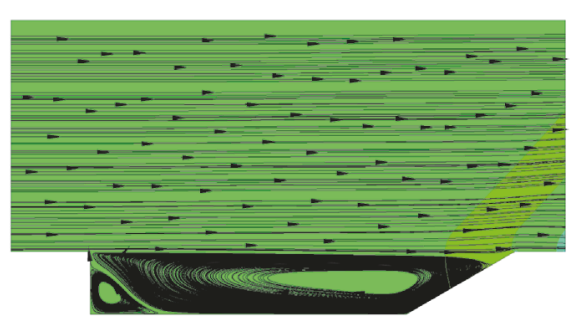

(e)

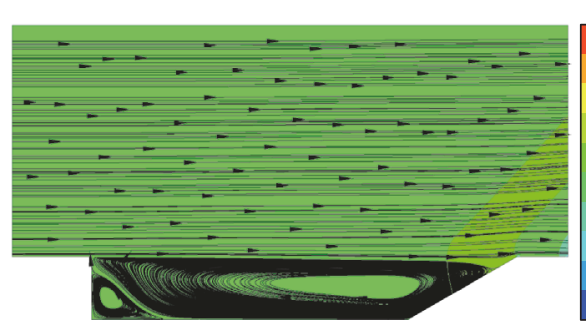

(g)

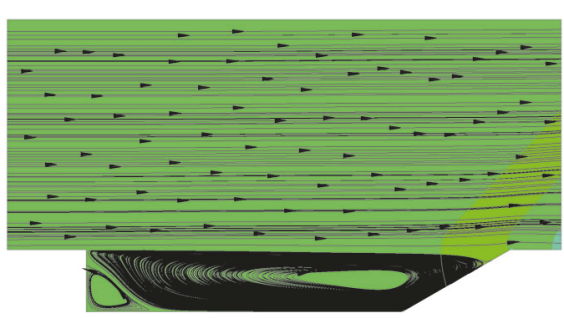

(i)

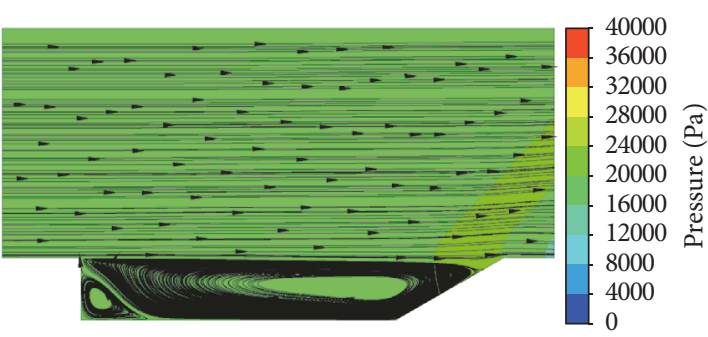

(b)

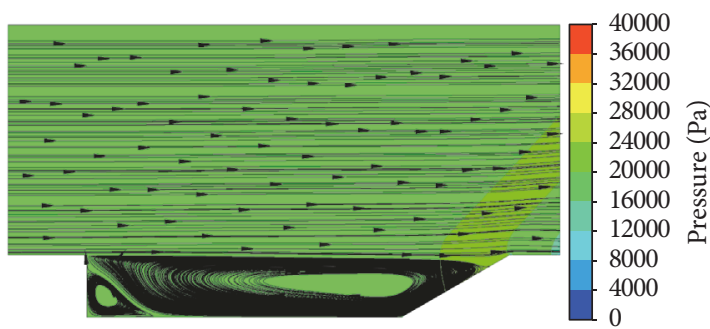

(d)

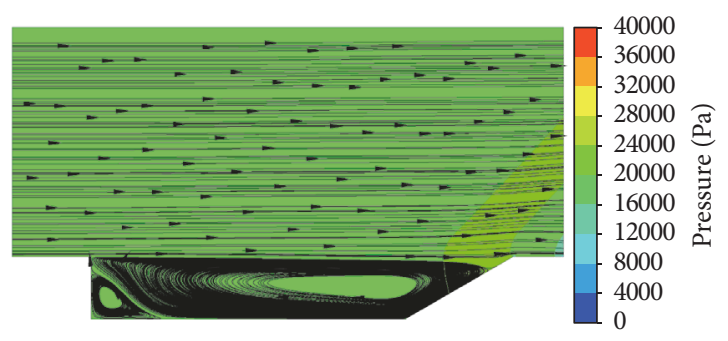

(f)

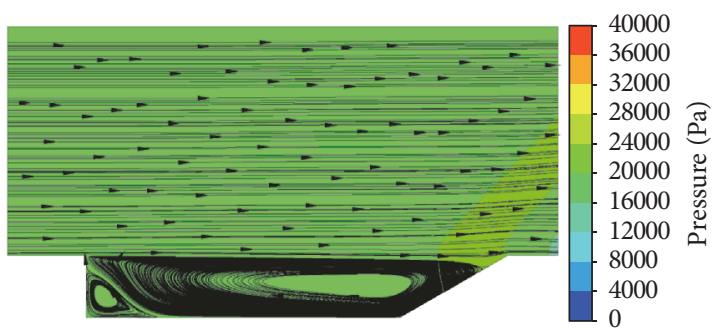

(h)

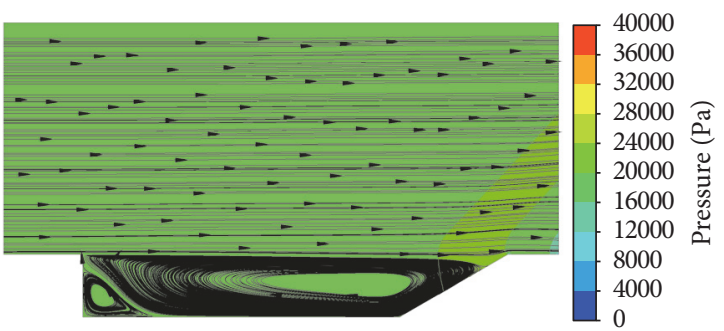

(j)

Figure 20: Pressure fluctuations of the whole domain (TWM3) (a) $t / t_{R}=0$, (b) $t / t_{R}=0.11$, (c) $t / t_{R}=0.22$, (d) $t / t_{R}=0.33$, (e) $t / t_{R}=0.44$, (f) $t / t_{R}=0.55$, (g) $t / t_{R}=0.66$, (h) $t / t_{R}=0.77$, (i) $t / t_{R}=0.88$, and (j) $t / t_{R}=1$.

Air is blown through the holes from outside to inside. Table 6 shows the details of the cases.

The results for the sound pressure levels are shown for the cavity walls in Figure 29. It is seen from the graphs that active control is not as effective as passive control techniques since it only provides around $5 \mathrm{~dB}$ of decrease in pressure fluctuations. However, active control methods have an inherent advantage of being able to adapt to changing conditions, 

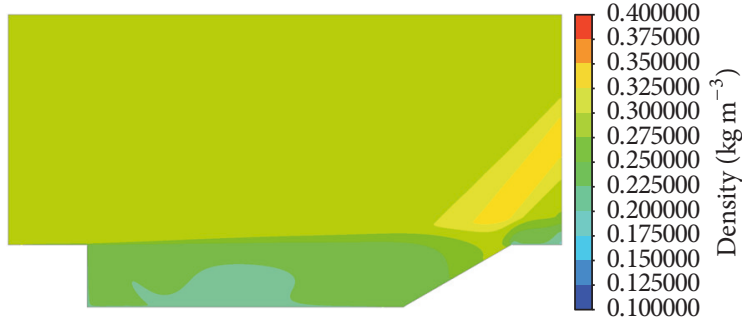

(a)
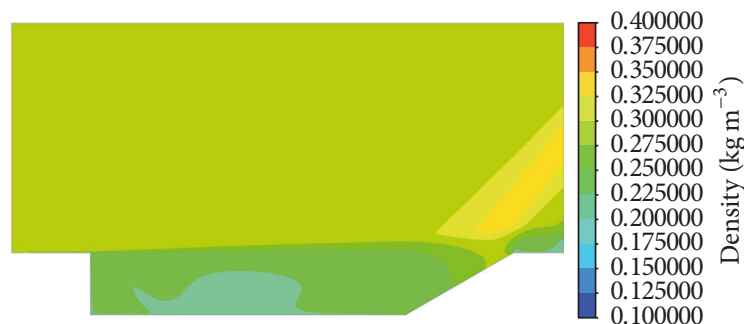

(c)
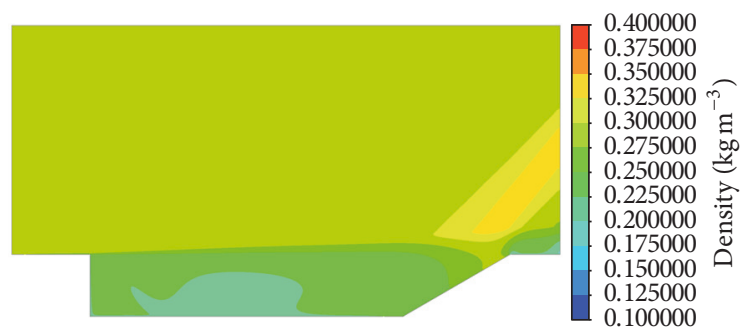

(e)

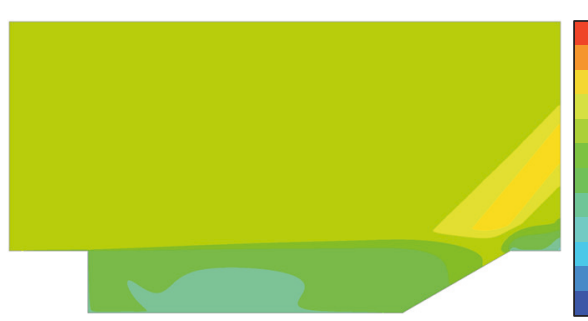

0.400000 0.375000 0.350000 0.325000 ? 0.275000 .250000 0.225000 0.200000 0.175000 0.150000 0.125000 0.125000

(g)

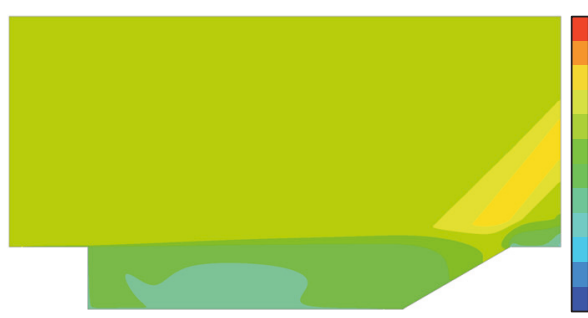

(i)

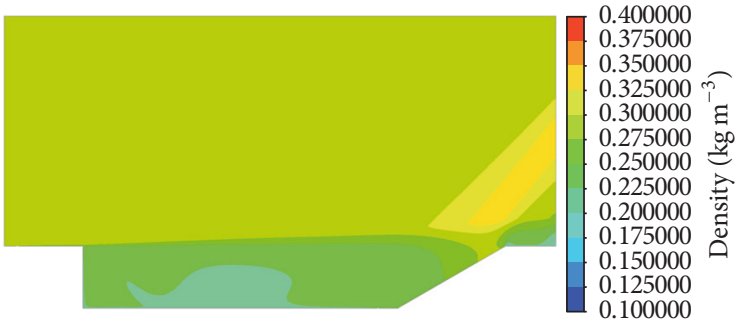

(b)

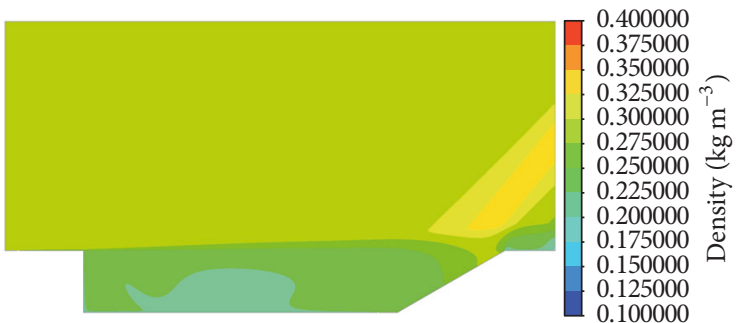

(d)

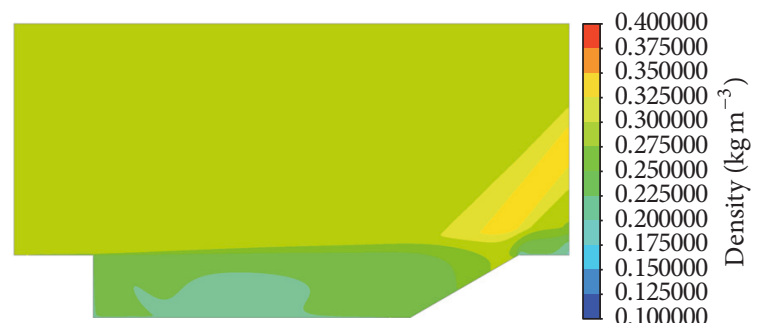

(f)

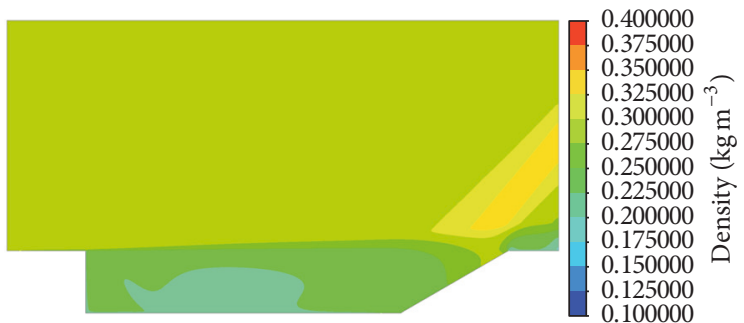

(h)

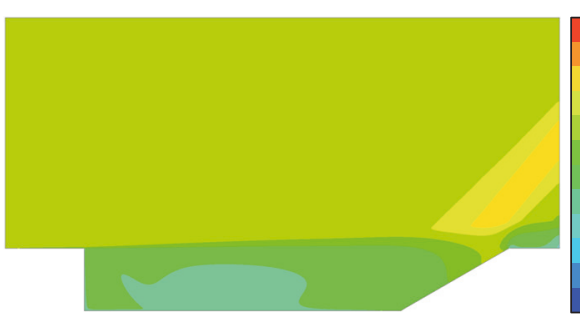

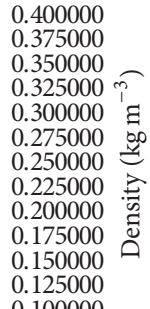

(j)

Figure 21: Density contours for TWM3 case (a) $t / t_{R}=0$, (b) $t / t_{R}=0.11$, (c) $t / t_{R}=0.22$, (d) $t / t_{R}=0.33$, (e) $t / t_{R}=0.44$, (f) $t / t_{R}=0.55$, (g) $t / t_{R}=0.66$, (h) $t / t_{R}=0.77$, (i) $t / t_{R}=0.88$, and (j) $t / t_{R}=1$.

whereas passive control methods are more effective; but they are not adjustable. Once implemented, they cannot be changed during flight.

3.7. Comparison of the Most Effective Cases of Each Control Method. In this part of the article, the results of the best cases of each control method are compared with the results of other control methods. The best cases are chosen as the most effective cases where the magnitude and frequency of the pressure oscillations decrease. Figure 30 shows the pressure oscillations for the selected control cases for the control point. As clearly observed from the graph, the magnitude of the 


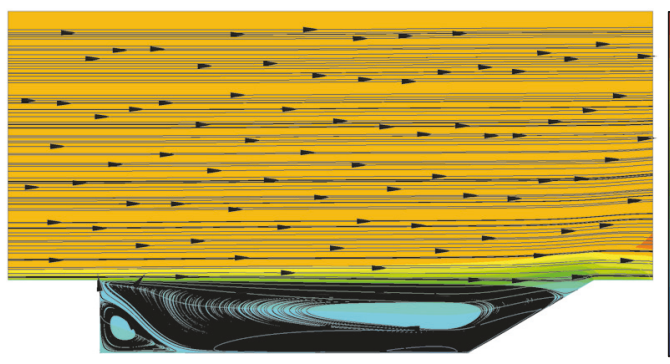

(a)

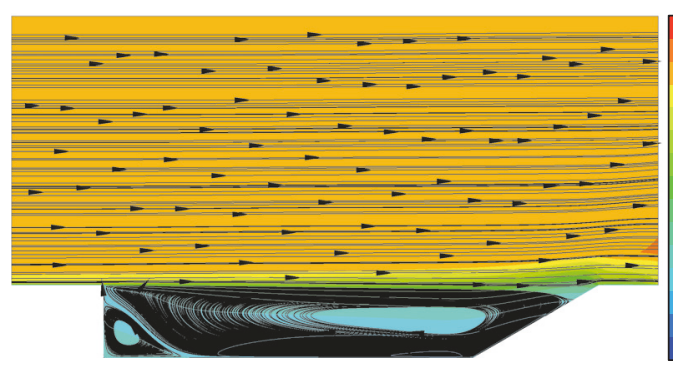

(c)

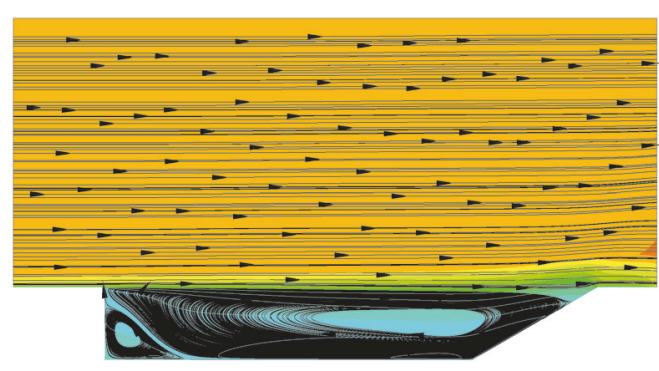

(e)

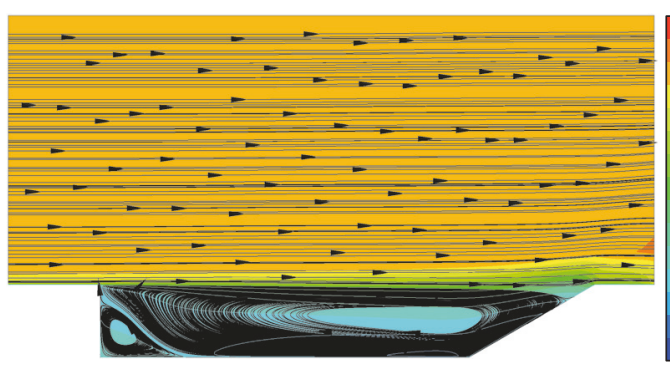

(g)

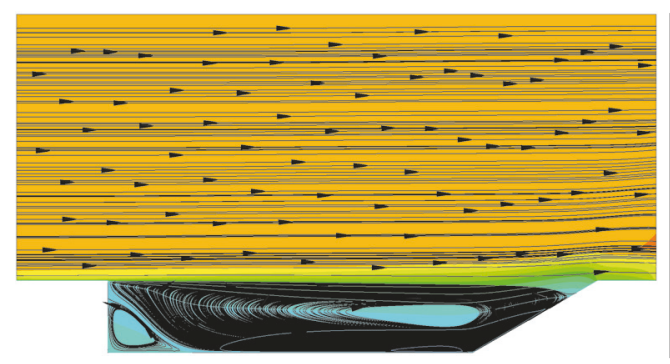

(i)
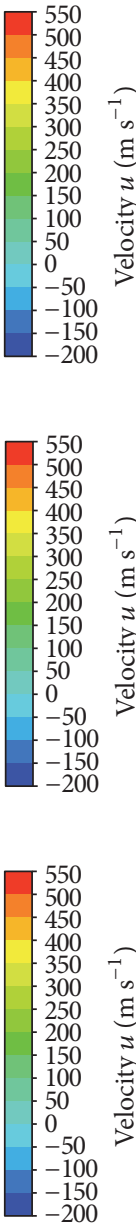

$-200$
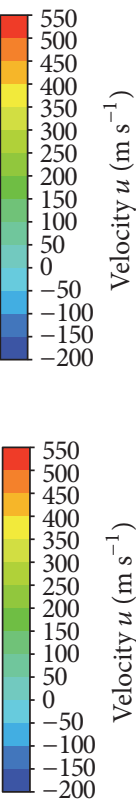

$-200$
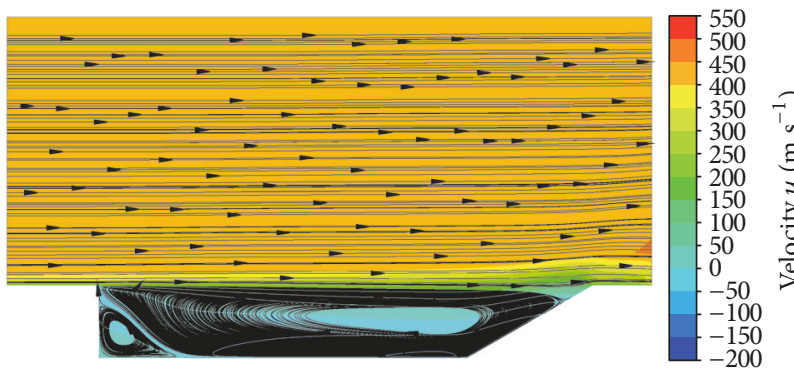

(b)
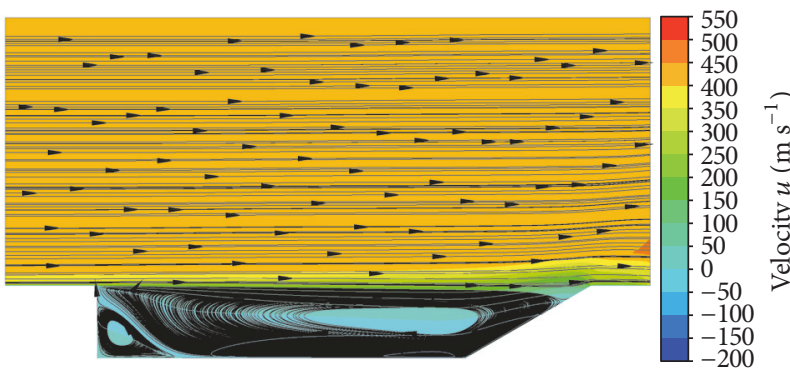

(d)
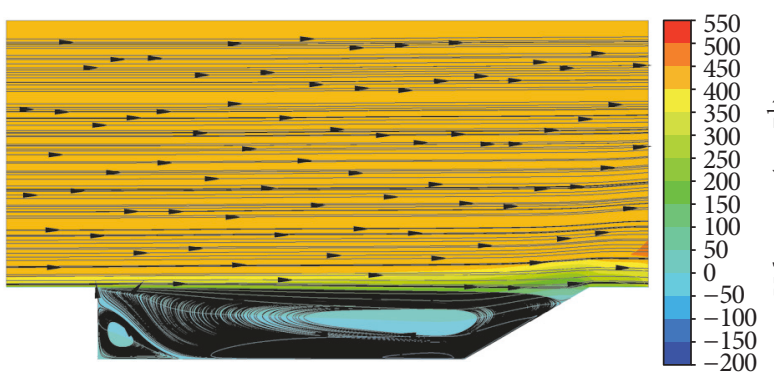

(f)
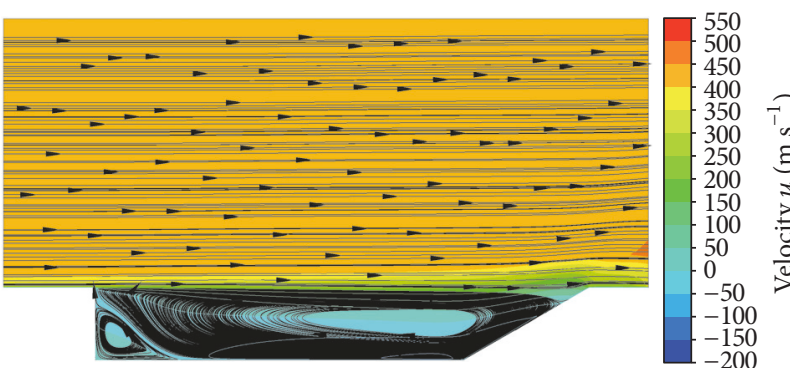

(h)
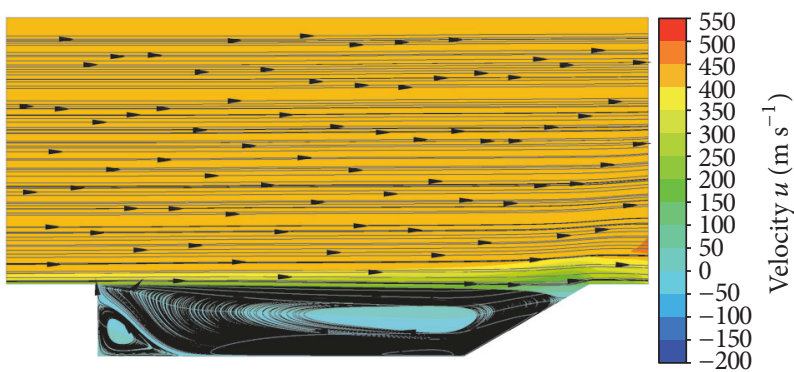

(j)

Figure 22: $X$-velocity contours and streamlines for TWM3 case (a) $t / t_{R}=0$, (b) $t / t_{R}=0.11$, (c) $t / t_{R}=0.22$, (d) $t / t_{R}=0.33$, (e) $t / t_{R}=0.44$, (f) $t / t_{R}=0.55$, (g) $t / t_{R}=0.66$, (h) $t / t_{R}=0.77$, (i) $t / t_{R}=0.88$, and (j) $t / t_{R}=1$. 


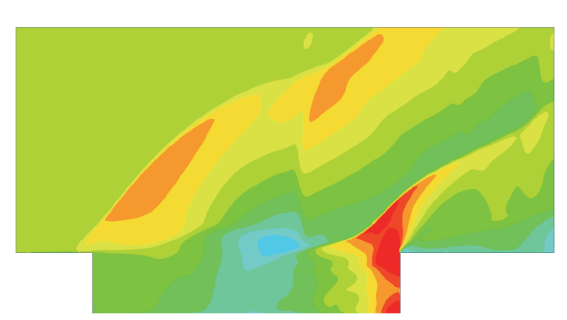

(a)

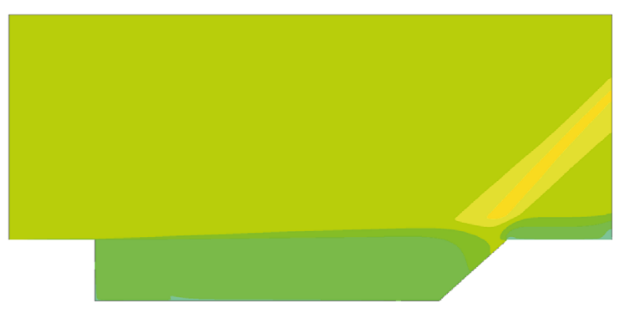

(c)

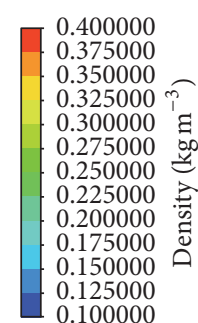

0.400000 0.375000 0.350000 0.325000 ? 0.275000 ? 0.250000 0.225000 0.200000 0.175000 ๑ 0.150000 0.125000 0.100000

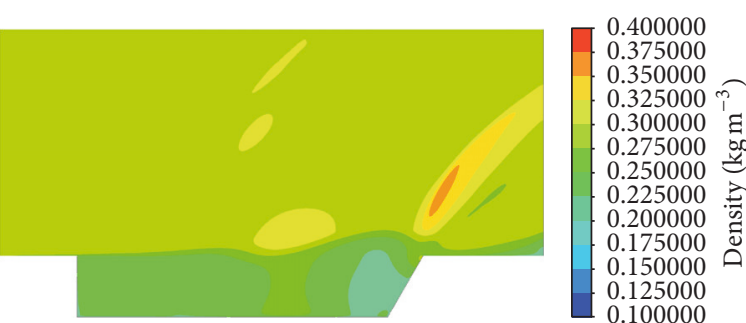

(b)

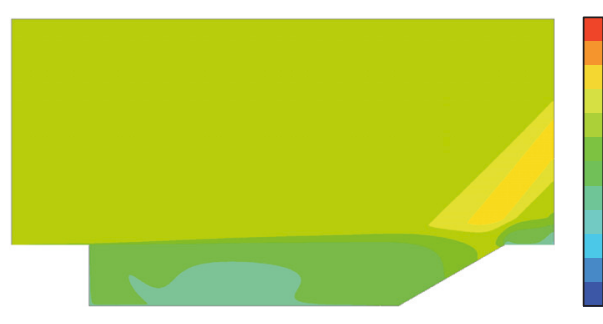

0.400000 0.375000 $0.350000=$ 0.325000 ? 0.300000 घ 0.275000 우 0.250000 (2) 0.225000 0.200000 0.175000 0.150000 0.125000 0.100000

(d)

FIGURE 23: Density contours for baseline and trailing edge wall modification cases $\left(t / t_{R}=0.55\right.$ ) (a) baseline, (b) TWM 1, (c) TWM 2, and (d) TWM 3.

TABLE 6: Jet blowing cases.

\begin{tabular}{lcccc}
\hline Case & T1 & T2 & L1 & L2 \\
\hline JB 1 & Open & Closed & Closed & Closed \\
JB 2 & Open & Open & Closed & Closed \\
JB 3 & Closed & Closed & Open & Open \\
JB 4 & Closed & Closed & Open & Open \\
JB 5 & Open & Open & Open & \\
\hline
\end{tabular}

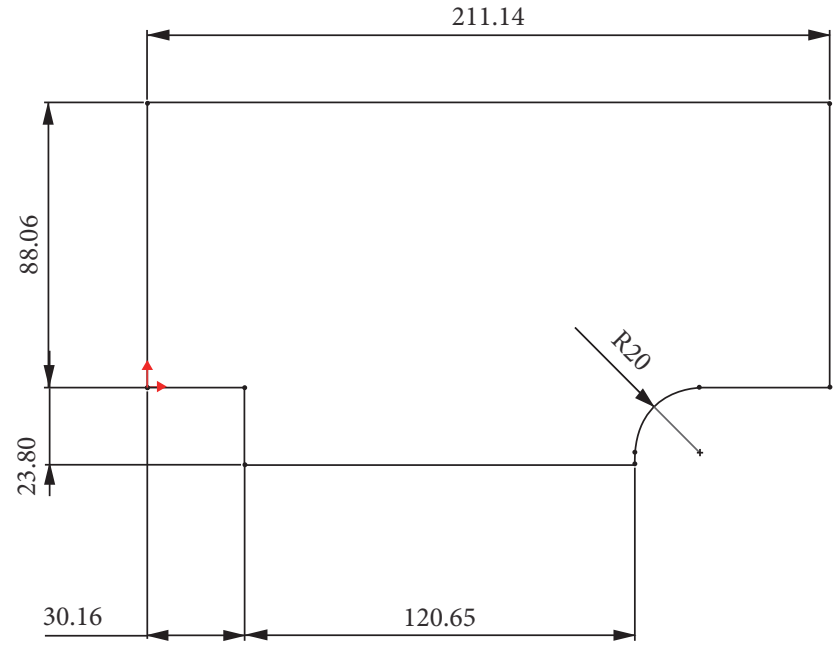

FIGURE 24: Cavity geometry with a curved trailing edge.

pressure oscillations decreases with jet blowing; however the oscillations are still periodic. The cover plate decreases the magnitude of the pressure oscillations drastically since it disrupts the feedback loop of the oscillations by providing a physical barrier between the inside and outside of the cavity,

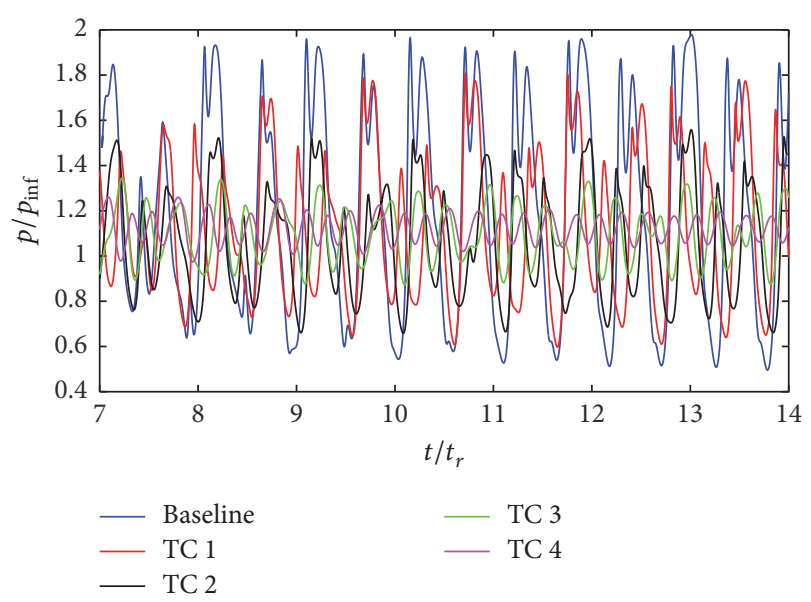

FIgURE 25: Pressure distribution for the control point for the cases with passive control with trailing edge wall curvature.

which also disturbs the free stream flow. When a curvature is given to the trailing edge wall, again, the magnitudes of the pressure oscillations decrease, still providing periodic flow, however, smoothening the flow. On the contrary, altering the trailing edge wall changes the periodic nature of the 


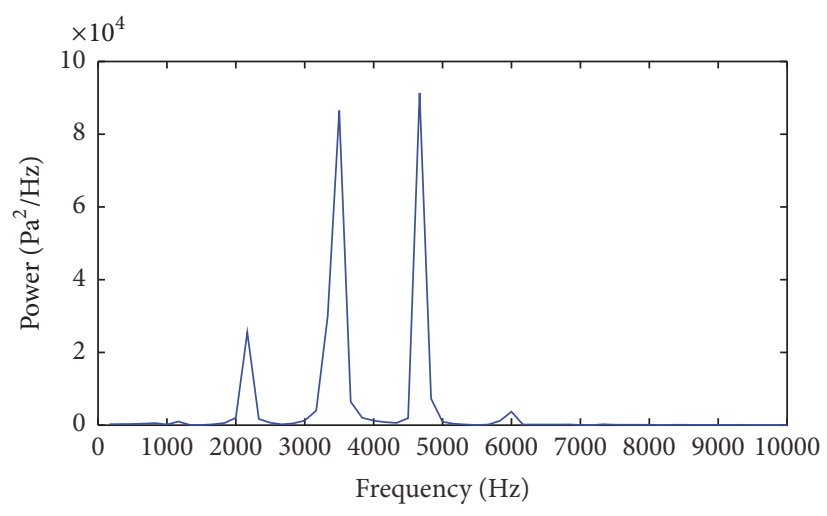

FIGURE 26: FFT of pressure for the control point (TC3).

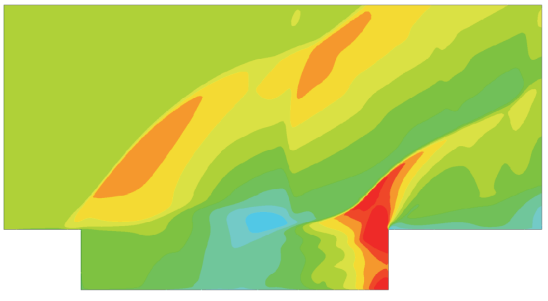

(a) Baseline

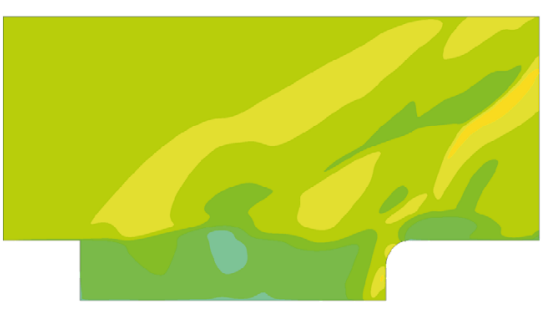

(c) TC2

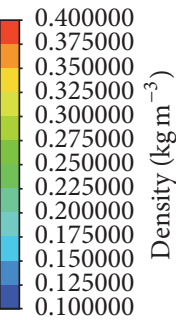

0.100000

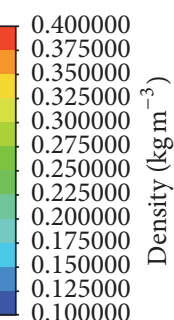

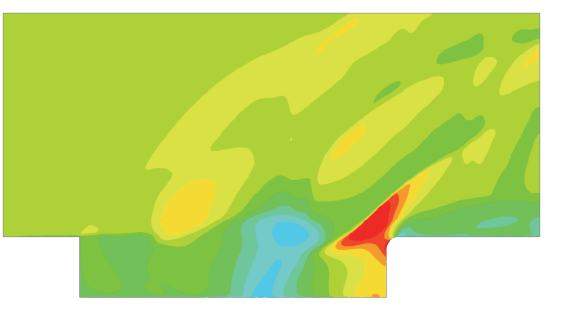

(b) TCl

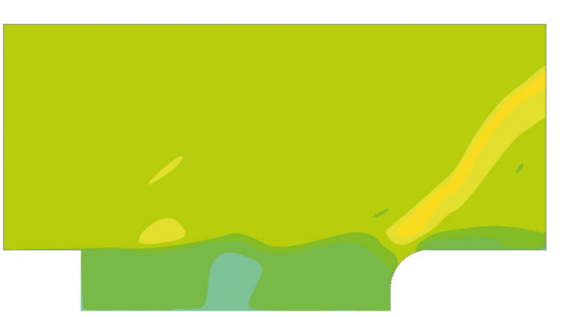

(d) TC3

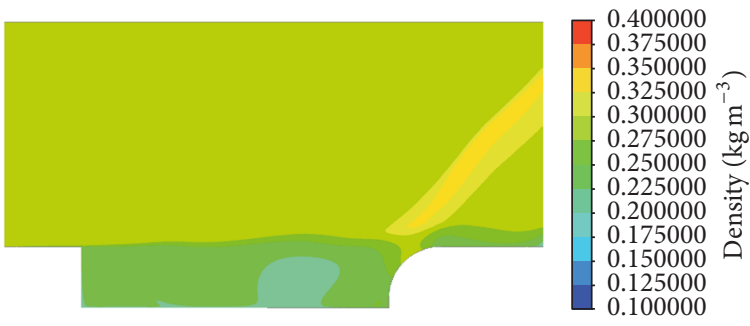

(e) TC4

Figure 27: Density contours for baseline and trailing edge curvature cases $\left(t / t_{R}=0.55\right)$.

cavity flow. All these control methods prove to be effective in suppressing pressure oscillations.

Table 7 shows the average reductions in sound pressure levels for the cavity walls. As seen in the table, when the trailing edge wall is modified the sound pressure levels for the cavity walls can be increased up to $40 \mathrm{~dB}$. Cover plate and trailing edge wall curvature are also effective methods that can decrease the SPL levels by $20 \mathrm{~dB}$. Although jet blowing does not seem as effective as the passive control methods, a $5 \mathrm{~dB}$ decrease is also high in terms of active flow control, since the flow is not irreversibly modified with active methods as in passive control techniques.

\section{Discussion and Conclusions}

Active and passive control techniques are utilized to suppress the pressure oscillations inside cavities caused by the supersonic flow past them. Jet blowing at several locations on the front and aft walls of the cavity configuration is used as an active control method. Several techniques are used for 


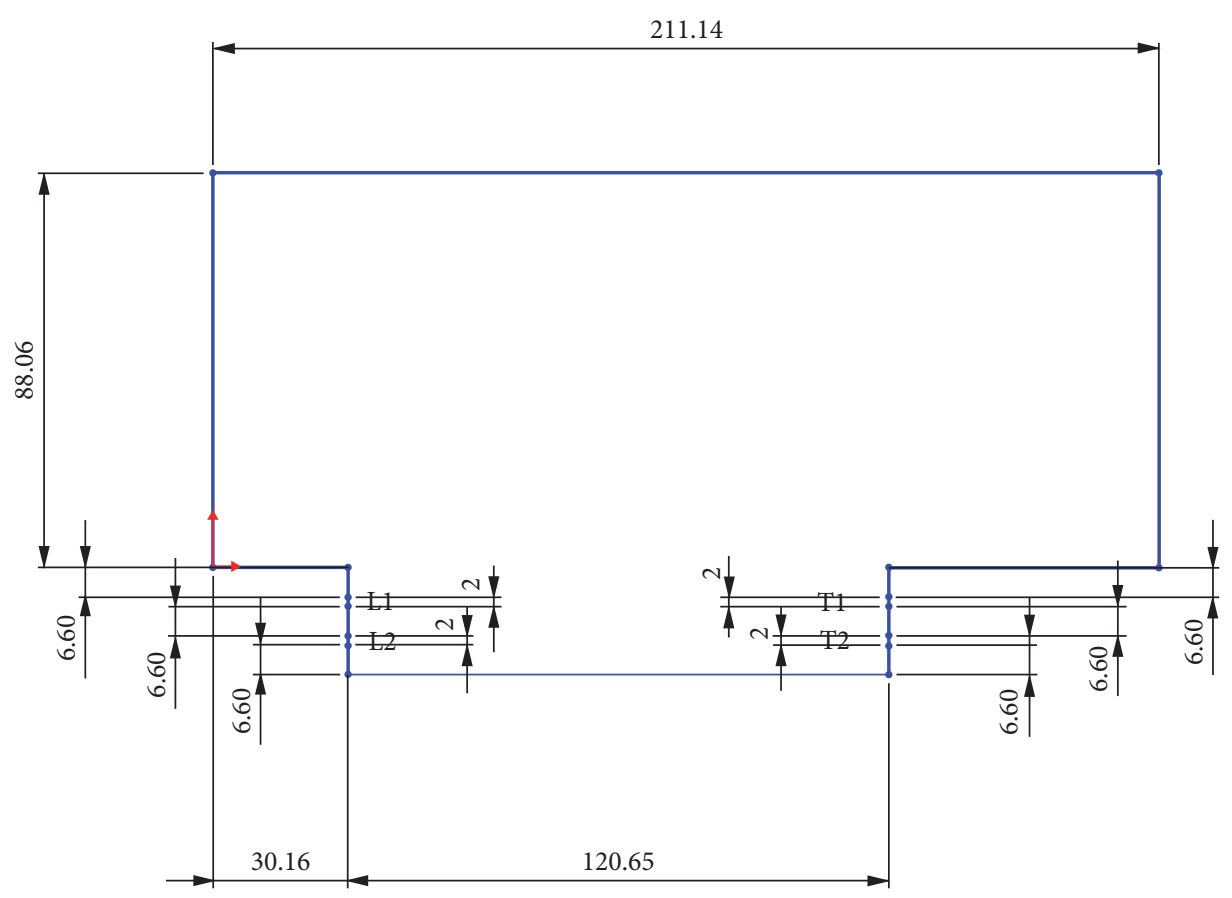

FIgURE 28: Location of holes for jet blowing.
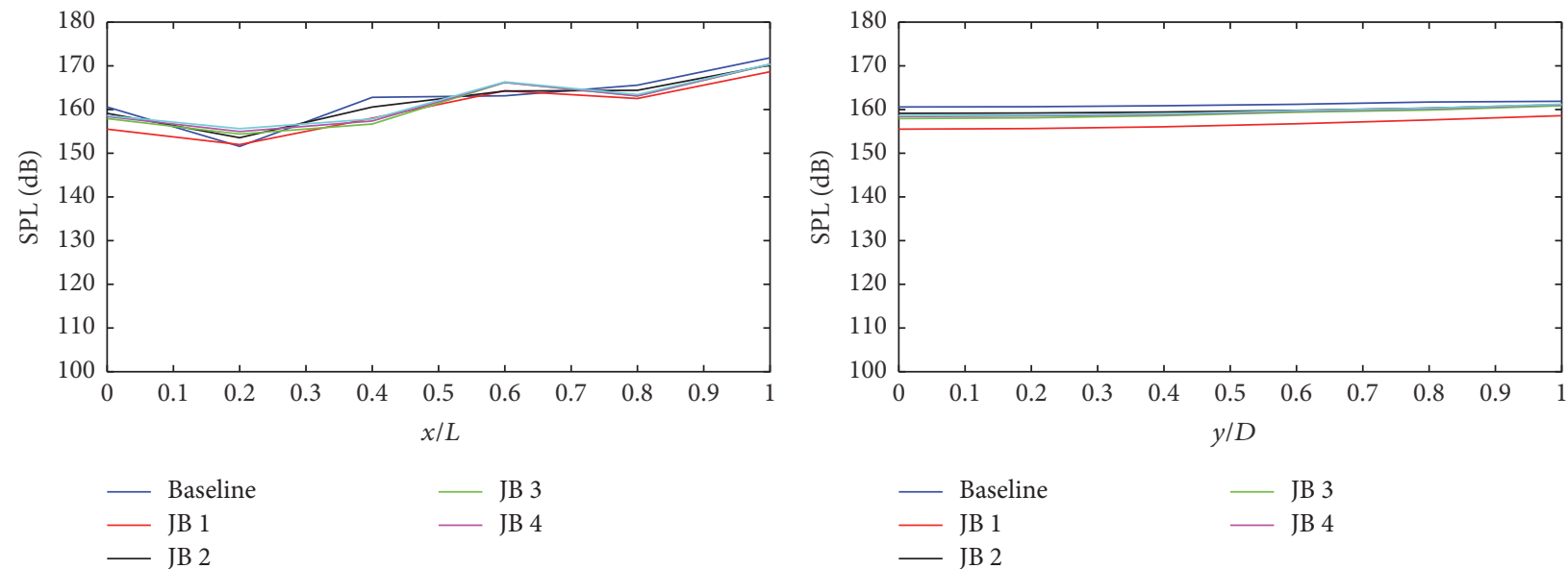

(a)

- JB 2

(b)

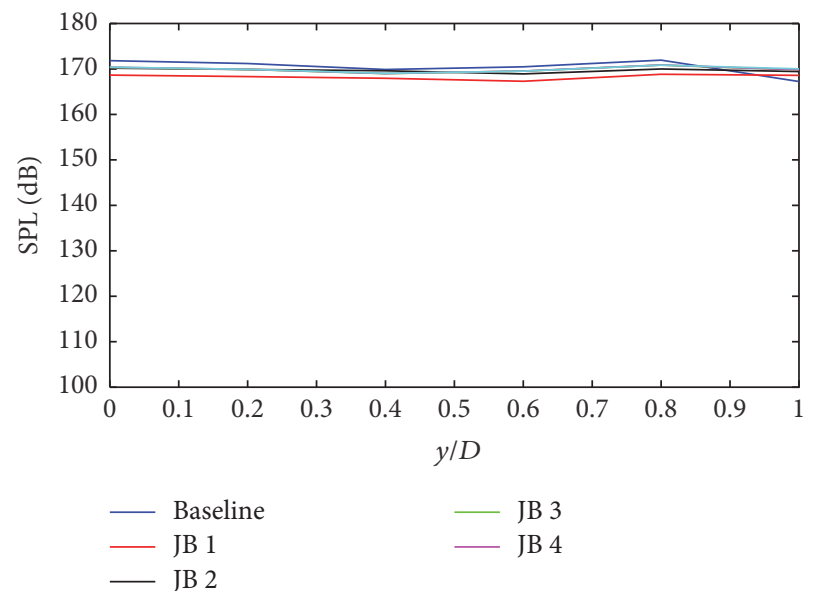

(c)

FIGURE 29: SPL values for (a) bottom wall, (b) leading edge wall, and (c) trailing edge wall for active control cases. 
TABLE 7: Comparison of sound pressure levels for the most effective cases and the baseline.

\begin{tabular}{|c|c|c|c|c|c|c|}
\hline Method & Bottom wall average SPL (dB) & $\%$ reduction & LE wall average SPL $(\mathrm{dB})$ & $\%$ reduction & TE wall average SPL (dB) & $\%$ reduction \\
\hline Baseline & 162.6 & - & 161.2 & - & 170.4 & - \\
\hline $\mathrm{CP} 4$ & 147.3 & 9.4 & 145.4 & 9.8 & 153.8 & 9.7 \\
\hline TWM 3 & 131.4 & 19.2 & 130.8 & 18.9 & 138.0 & 19.0 \\
\hline TC 4 & 141.2 & 13.2 & 135.4 & 16.0 & 150.3 & 11.8 \\
\hline JB1 & 160.2 & 1.5 & 156.7 & 2.8 & 168.3 & 1.2 \\
\hline
\end{tabular}

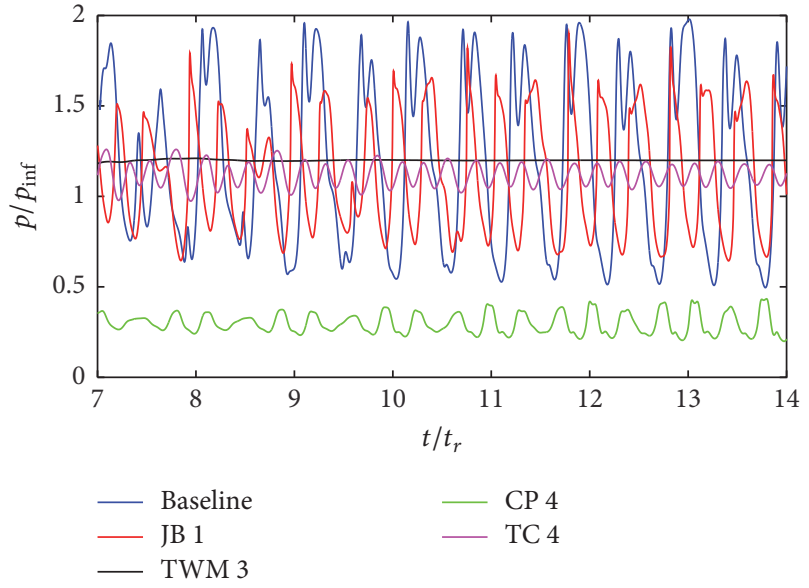

Figure 30: Pressure distribution comparison for the most effective control cases.

passive control including, using a cover plate to separate the flow dynamics inside and outside of the cavity, trailing edge wall modifications, such as inclination of the trailing edge, and providing curvature to the trailing edge wall. The results of active and passive control techniques are compared with the baseline case in terms of pressure fluctuations, sound pressure levels at the leading edge, trailing edge walls, and cavity floor and in terms of formation of flow structures as observed from pressure, density contours, and $x$-velocity contours superimposed with streamlines.

Per the results presented, it is shown that the most effective passive control method is the modification of the trailing edge wall among all the methods tested. Approximately $20 \%$ reduction is observed for the average sound pressure levels on the cavity walls including the trailing edge cavity wall. When the pressure fluctuations are examined, it is seen that the periodic nature of the flow is eliminated when the trailing edge wall is modified by introducing a slope. Changing the curvature of the trailing edge wall is also effective, although it is not as effective as introducing a slope. The average SPL values for the cavity walls decrease by $12-13 \%$ with this method.

Introducing a cover plate from the leading edge of the cavity is also effective in suppressing the oscillations inside the cavity. $10 \%$ reduction in sound pressure levels is observed with a cover plate, by altering the slope of the plate. The plate separates the inside and outside of the cavity, thereby leading to two separate zones for the formation of the vortices inside.

As an active control method, jet blowing is introduced from several locations on the cavity walls. Most effective jet blowing configuration is blowing from the trailing edge wall with $10 \%$ of the free stream velocity among the cases that are numerically tested. Active control is not as effective as passive control techniques since it only provides around $5 \mathrm{~dB}$ of decrease in pressure fluctuations. However, active control methods have an inherent advantage of being able to adapt to changing conditions, whereas passive control methods are more effective; but they are not adjustable. Once implemented, they cannot be changed during flight.

This study shows that supersonic cavity flow pressure oscillations which is crucial for weapon bay aerodynamics can be suppressed with the help of active and passive control techniques if they are properly applied to the geometry.

\section{Conflicts of Interest}

The authors declare that there are no conflicts of interest regarding the publication of this paper.

\section{References}

[1] S. Aradag, H. Kim, and D. D. Knight, "Two and three dimensional simulations of supersonic cavity flow," Engineering Applications of Computational Fluid Mechanics, vol. 4, no. 4, pp. 612-621, 2010.

[2] S. J. Lawson and G. N. Barakos, "Review of numerical simulations for high-speed, turbulent cavity flows," Progress in Aerospace Sciences, vol. 47, no. 3, pp. 186-216, 2011.

[3] L. N. Cattafesta III, Q. Song, D. R. Williams, C. W. Rowley, and F. S. Alvi, "Active control of flow-induced cavity oscillations," Progress in Aerospace Sciences, vol. 44, no. 7-8, pp. 479-502, 2008.

[4] D. P. Rizzetta, M. R. Visbal, and P. E. Morgan, "A high-order compact finite-difference scheme for large-eddy simulation of active flow control," Progress in Aerospace Sciences, vol. 44, no. 6, pp. 397-426, 2008.

[5] P. Comte, F. Daude, and I. Mary, "Simulation of the reduction of unsteadiness in a passively controlled transonic cavity flow," Journal of Fluids and Structures, vol. 24, no. 8, pp. 1252-1261, 2008.

[6] V. Levasseur, P. Sagaut, M. Mallet, and F. Chalot, "Unstructured Large Eddy Simulation of the passive control of the flow in a weapon bay," Journal of Fluids and Structures, vol. 24, no. 8, pp. 1204-1215, 2008.

[7] S. Arunajatesan, C. Kannepalli, N. Sinha et al., "Suppression of cavity loads using leading-edge blowing," AIAA Journal, vol. 47, no. 5, pp. 1132-1144, 2009.

[8] S. Aradag, H. Yan, and D. Knight, "The effects of laser energy deposition on supersonic cavity flow," Journal of Thermal Science and Technology, vol. 29, no. 2, pp. 67-73, 2009. 
[9] I. Yilmaz and S. Aradag, "Numerical laser energy deposition on supersonic cavity flow and sensor placement strategies to control the flow," The Scientific World Journal, vol. 2013, Article ID 141342, 8 pages, 2013.

[10] E. Lazar, G. Elliotts, and N. Glumac, "Control of the shear layer above a supersonic cavity using energy deposition," AIAA Journal, vol. 46, no. 12, pp. 2987-2997, 2008.

[11] Y. Lee, M. Kang, H. Kim, and T. Setoguchi, "Passive control techniques to alleviate supersonic cavity flow oscillation," Journal of Propulsion and Power, vol. 24, no. 4, pp. 697-703, 2008.

[12] L. Ukeiley, M. Sheehan, F. Coiffet, F. Alvi, S. Arunajatesan, and B. Jansen, "Control of pressure loads in geometrically complex cavities," Journal of Aircraft, vol. 45, no. 3, pp. 1014-1024, 2008.

[13] F. S. Alvi and L. N. Cattafesta III, "The art and science of flow control-case studies using flow visualization methods," European Physical Journal: Special Topics, vol. 182, no. 1, pp. 97112, 2010.

[14] N. Zhuang, F. S. Alvi, M. B. Alkislar, and C. Shih, "Supersonic cavity flows and their control," AIAA Journal, vol. 44, no. 9, pp. 2118-2128, 2006.

[15] V. Narayanaswamy, L. L. Raja, and N. T. Clemens, "Characterization of a high-frequency pulsed-plasma jet actuator for supersonic flow control," AIAA Journal, vol. 48, no. 2, pp. 297305, 2010.

[16] V. Thangamani and J. Kurian, "Control of cavity oscillations in a supersonic flow by microjet injection," Journal of Aircraft, vol. 50, no. 4, pp. 1305-1308, 2013.

[17] L. G. Kaufman, A. Maciulaitis, and R. L. Clark, "Mach 0.6 to 3.0 flows over rectangular cavities," Tech. Rep. AFWAL-TR-82-3112, Air Force Wright Aeronautical Labs, 1983.

[18] I. Yilmaz, E. Ayli, and S. Aradag, "Investigation of the effects of length to depth ratio on open supersonic cavities using CFD and proper orthogonal decomposition," The Scientific World Journal, vol. 2013, Article ID 810175, 12 pages, 2013.

[19] J. E. Rossiter, "Wind tunnel experiments on the flow over rectangular cavities at subsonic and transonic speeds," Tech. Rep. 64037, Royal Aircraft Establishment, Surrey, UK, 1964. 


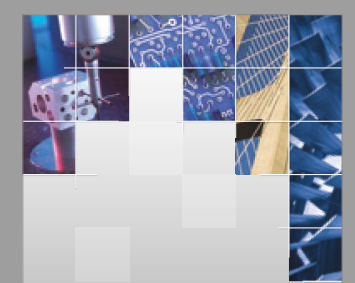

\section{Enfincering}
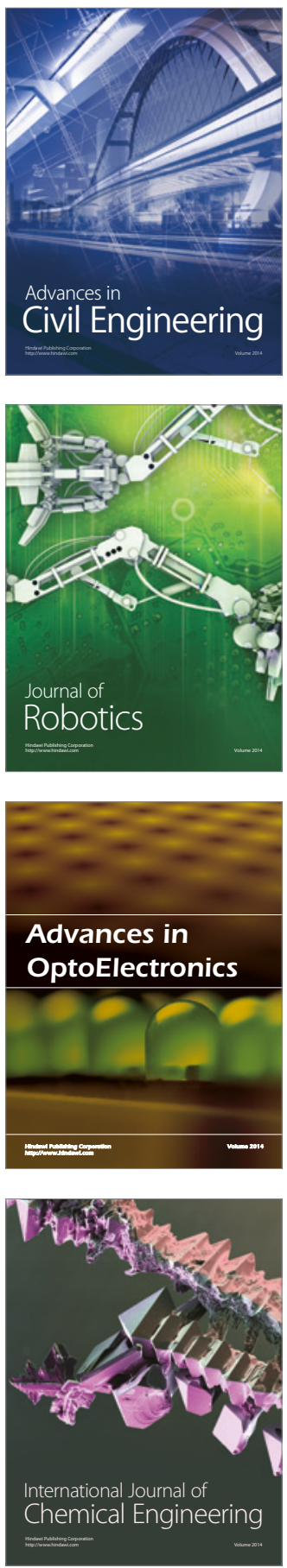

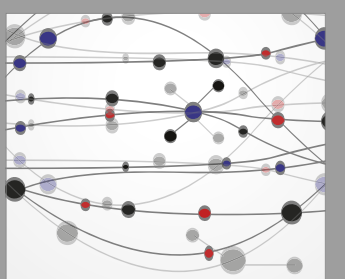

The Scientific World Journal

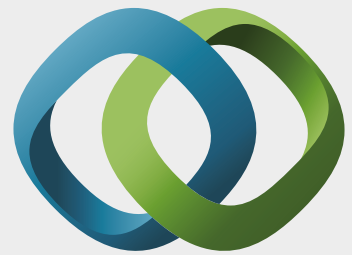

\section{Hindawi}

Submit your manuscripts at

https://www.hindawi.com
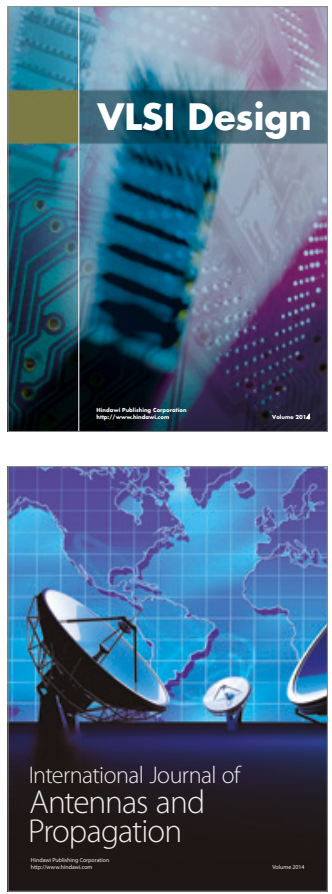

\section{Rotating}

Machinery
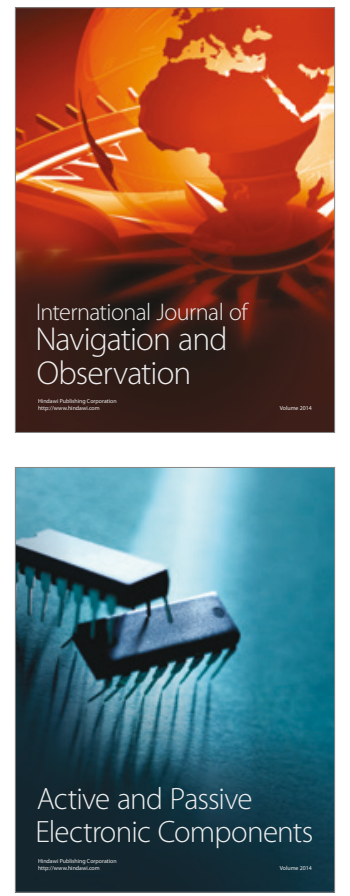
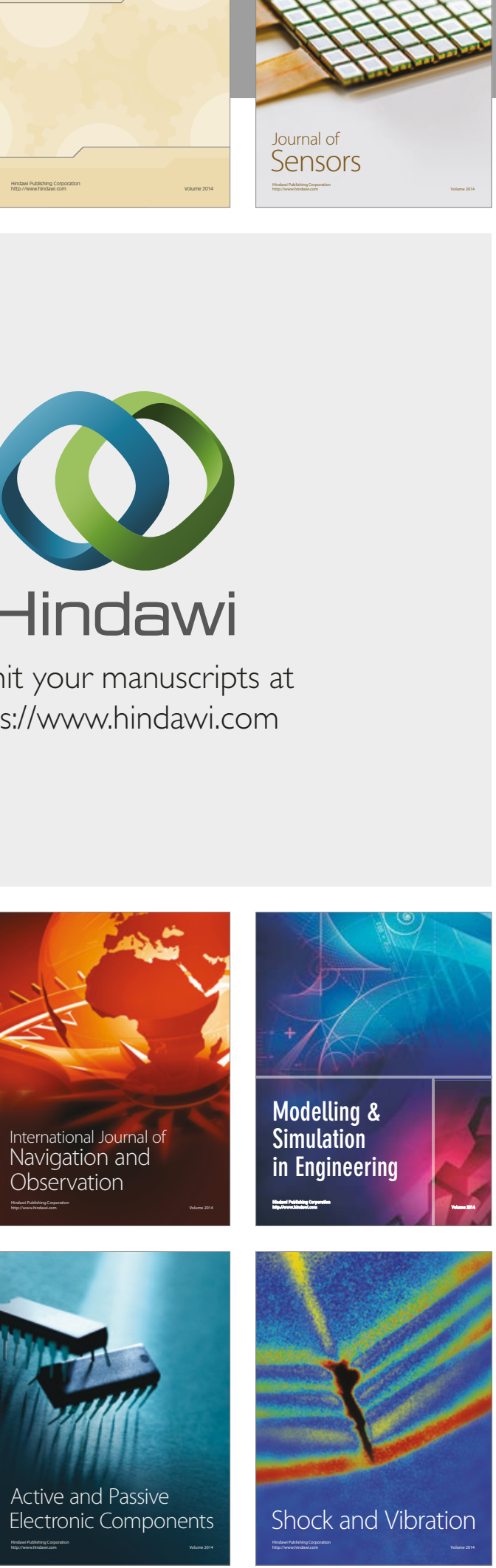
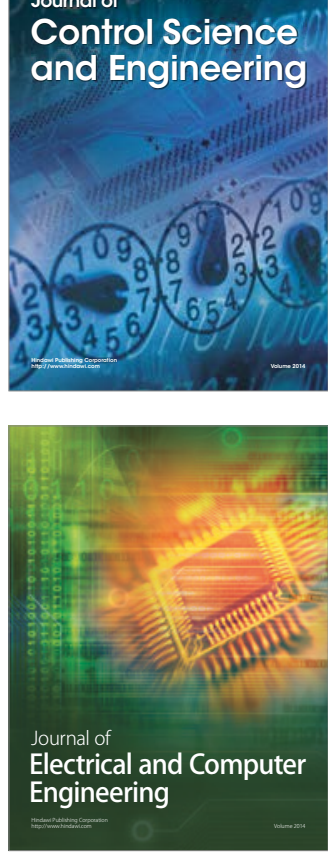

Distributed

Journal of

Control Science

and Engineering
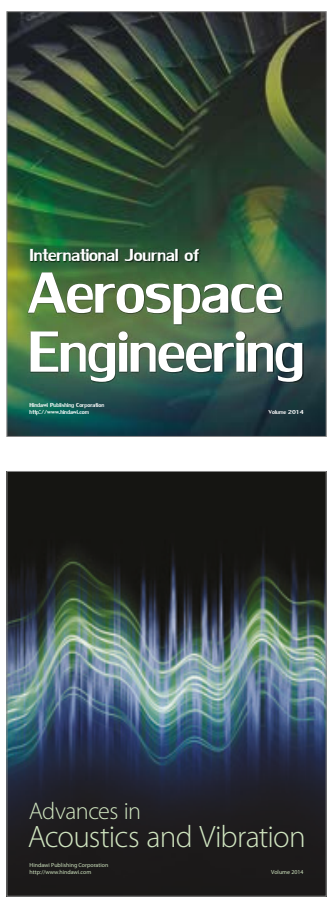

Sensor Networks 